Accelerator Division

Alternating Gradient Synchrotron Department

BROOKHAVEN NATIONAL LAABORATORY

Upton, New York 11973

Accelerator Division

Technical Note

AGS/AD/Tech. Note No. 356

SOLUTIONS OF LAPLACE'S EQUATION AND MULTIPOLE

EXPANSIONS WITH A CURVED LONGITUDINAL AXIS

S.R. Mane

November 21, 1991 


\title{
Solutions of Laplace's Equation and Multipole Expansions with a Curved Longitudinal Axis *
}

\author{
S.R. Mane \\ Brookhaven National Laboratory, Upton, N.Y. 11973.
}

\section{Contents}

1 Introduction

2 Laplace's Equation $\quad 2$

2.1 Multipole Expansion in Two Dimensions with Straight $z$ Axis . . . . . . . . 2

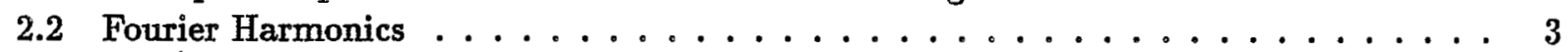

2.3 Equations for Scalar and Vector Potentials with Curved $z$ Axis . . . . . . . . . 4

2.4 General Expressions for Multipoles . . . . . . . . . . . . . . . . . 5

2.5 Tabulation of $U_{n}^{e, m}$ Functions ..................... . . 7

2.6 Recursion Relations for $U_{n}^{e, m}$ Functions $\ldots \ldots \ldots \ldots \ldots \ldots$

2.7 Multipoles for Electric and Magnetic Fields with Curved $z$ Axis . . . . . . . . . 12

2.8 Explicit Expressions for $U_{n}^{e, m}$ Functions . . . . . . . . . . . . . . . 13

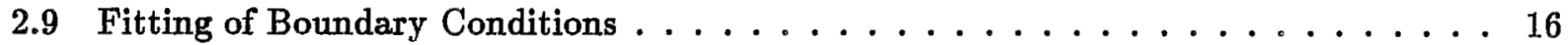

2.9 .1 General Procedure . . . . . . . . . . . . . . . . . 16

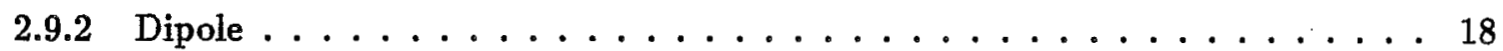

2.9.3 Torus with $\cos (2 \theta)$ Potential $\ldots \ldots \ldots \ldots \ldots$

2.9.4 Quadrupole Plates in Vacuum Chamber ................ 24

3 Conclusion $\quad 27$

$\begin{array}{lr}\text { Appendix } & 28\end{array}$

$\begin{array}{lr}\text { A Alternative Formalisms } & 28\end{array}$

A.1 Homogenous Polynomials . . . . . . . . . . . . . . . . . . 28

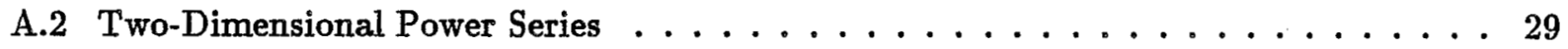

"Work performed under the auspices of the U.S. Department of Energy. 


\section{Introduction}

When maesuring the field in a magnet, such a combined function dipole, one usually attempts to decsribe the field as a sum of Fourier harmonics, or multipoles. There is a standard terminology of so-called "normal" and "skew" multipoles associated with this practice. The multipole content is useful to know for problems such as particle tracking, to determine orbit stability of the particles in a synchrotron or storage ring. In high-precision experiments such as the muon $g-2$ experiment at $B N L$, a knowledge of the field quality, i.e. multipoles, also affects the precision of the experiment, i.e. the measured value of $g-2$. The purpose of this report is to describe a set of functions which satisfy Laplace's equation in two dimensions, say $x$ and $y$. The functions do not depend on $z$; however the $z$ axis is not straight, but is curved in a circle of radius $\rho_{0}$. Hence the functions $d o$ depend on $\rho_{0}$, as well as $x$ and $y$. These functions are introduced to deal with problems, such as the fitting of magnetic field measurements, where the field is two dimensional, but the $z$ axis is curved. Typically, if the $z$ axis is straight, the field, or potential, can be expressed as a sum of Fourier harmonics $r^{n} \cos (n \theta)$ and $r^{n} \sin (n \theta)$, where $x=r \cos \theta$ and $y=r \sin \theta$. However, Fourier harmonics do not satisfy Laplace's equation in two dimensions when the $z$ axis is curved. The functions to be described below are therefore generalizations of the Fourier multipoles above. I shall refer to them as "curved multipoles."

Note that the term "curved longitudinal axis" or "curved $z$ axis" is ambiguous. Consider a rectangular dipole: we can draw a straight line through the middle, and define this line to be the $z$ axis. Then we can use Fourier harmonics to give a multipole decomposition of the field, relative to this axis. However, this is not the design orbit of the accelerator; it is not the axis the particles will follow. If we use that axis, which is a circular arc, then we shall need to use a more complicated representation of the field, specifically, I propose the use of curved multipoles. For a combined function dipole, one has to specify the higher order terms in the field to have a well-defined description of the magnetic field, and one is forced to introduce some generalization of the Fourier harmonics anyway. Note, however, that the prescription I shall offer below to derive the generalizations of the Fourier harmonics is not unique; other methods will be briefly described in the appendix.

\section{Laplace's Equation}

\subsection{Multipole Expansion in Two Dimensions with Straight $z$ Axis}

When the electric field is contained in the two-dimensional $(x, y)$ plane, it can be derived from a scalar potential $V=V(x, y)$, a function of $x$ and $y$ only. A magnetic field which is contained in the two-dimensional $(x, y)$ plane can be derived from a vector potential with only one nonzero component, i.e. $\vec{A}=A_{\phi} \mathbf{e}_{\phi}$, where $A_{\phi}=A_{\phi}(x, y)$, and $\mathbf{e}_{\phi}$ is a unit vector in the longitudinal direction. We shall usually call this the $z$ direction. To save on notation, we shall frequently use the symbol $\Phi$, or $\Phi^{e, m}$, to denote either $V$ or $A_{\phi}$, because many of the statements below will apply 
equally well to both $V$ and $A_{\phi}$.

When the $z$ axis is straight, Laplace's equation for both $V$ and $A_{\phi}$ has the common form

$$
\frac{\partial^{2} \Phi}{\partial x^{2}}+\frac{\partial^{2} \Phi}{\partial y^{2}}=0
$$

where, as stated above, $\Phi$ could denote either $V$ or $A_{\phi}$. It is well-known that $\Phi$ can be decomposed into a linear combination of so-called normal and skew multipoles, given by the general formula

$$
\sum_{n=1}^{\infty} \Phi_{n}^{n}+i \Phi_{n}^{s}=\sum_{n=1}^{\infty}(x+i y)^{n}=\sum_{n=1}^{\infty} r^{n} e^{i n \theta}=\sum_{n=1}^{\infty} r^{n}[\cos (n \theta)+i \sin (n \theta)],
$$

where the superscripts denote normal and skew multipoles, with an obvious notation. We see that $\Phi_{n}^{n}=r^{n} \cos (n \theta)$ and $\Phi_{n}^{s}=r^{n} \sin (n \theta)$. In Cartesian coordinates, the expressions for the first few multipoles are

$$
\begin{array}{ll}
\Phi_{1}^{n}=x, & \Phi_{1}^{s}=y, \\
\Phi_{2}^{n}=x^{2}-y^{2}, & \Phi_{2}^{s}=2 x y, \\
\Phi_{3}^{n}=x^{3}-3 x y^{2}, & \Phi_{3}^{s}=3 x^{2} y-y^{3}, \\
\Phi_{4}^{n}=x^{4}-6 x^{2} y^{2}+y^{4}, & \Phi_{4}^{s}=4 x^{3} y-4 x y^{3}, \\
\Phi_{5}^{n}=x^{5}-10 x^{3} y^{2}+5 x y^{4}, & \Phi_{5}^{s}=5 x^{4} y-10 x^{2} y^{3}+y^{5}, \\
\Phi_{6}^{n}=x^{6}-15 x^{4} y^{2}+15 x^{2} y^{4}-y^{6}, & \Phi_{6}^{s}=6 x^{5} y-20 x^{3} y^{3}+6 x y^{5} .
\end{array}
$$

The general formula for the coefficients is

$$
\begin{aligned}
\Phi_{n}^{n} & =\sum_{k=0}^{K_{1}}(-1)^{k} \frac{n !}{(n-2 k) !(2 k) !} x^{n-2 k} y^{2 k}, \\
\Phi_{n}^{s} & =\sum_{k=0}^{K_{2}}(-1)^{k} \frac{n !}{(n-2 k-1) !(2 k+1) !} x^{n-2 k-1} y^{2 k+1},
\end{aligned}
$$

where $K_{1}$ and $K_{2}$ are the largest integers such that $n-2 k \geq 0$ and $n-2 k-1 \geq 0$, respectively. Hence $K_{1}=n / 2, K_{2}=(n-2) / 2$ for even $n$ and $K_{1}=K_{2}=(n-1) / 2$ for odd $n$.

\subsection{Fourier Harmonics}

Given a potential $\Phi^{e, m}(x, y)$ in some region of space which contains no charges, we can decompose it into a sum of multipoles. The procedure is well-known. We integrate $\Phi^{e, m}$ around a circle of radius $r_{n o r m}$, and calculate

$$
a_{n} \equiv \oint\left[\Phi^{e, m}(x, y)\right]_{r=r_{n o r m}} \sin (n \theta) \frac{d \theta}{2 \pi}
$$




$$
b_{n} \equiv \oint\left[\Phi^{e, m}(x, y)\right]_{r=r_{n o r m}} \cos (n \theta) \frac{d \theta}{2 \pi},
$$

for $n \geq 1$, where $x=r \cos \theta$ and $y=r \sin \theta$. The above are definitions of $a_{n}$ and $b_{n}$. The potential, when represented in the form $\Phi^{e, m}=\Phi^{e, m}(r, \theta)$ is defined to be

$$
\Phi^{e, m}(r, \theta) \equiv \sum_{n=1}^{\infty}\left(\frac{r}{r_{n o r m}}\right)^{n}\left[b_{n} \cos (n \theta)+a_{n} \sin (n \theta)\right] \equiv \sum_{n=1}^{\infty} B_{n} r^{n} \cos (n \theta)+A_{n} r^{n} \sin (n \theta) .
$$

The coefficients $A_{n}$ and $B_{n}$ have units of Volts $/\left(\right.$ meter) ${ }^{n}$, or Tesla $/\left(\right.$ meter) ${ }^{n}$, as appropriate, as opposed to $a_{n}$ and $b_{n}$ which have units of Volts or Tesla for all $n$. For numerical work the upper limit on the sum is of course finite. It is well-known that Eq. (2.6) is the general solution for $\Phi^{e, m}$ in two dimensions, when (1) no charges are enclosed in the domain of the multipole expansion, and (2) the $z$ axis is straight. One can also add a $B_{0}$ term, but that is a constant, and is neglected by programs such as PoIsson because an additive constant does not contribute to the field.

The above statements explain the reason for the standard multipole expansion in Fourier harmonics: it yields the general form of the solution of Laplace's equation in two dimensions, within certain well-defined circumstances. However, when the $z$ axis is curved in a circle, Eq. (2.6) is not the general solution for $\Phi^{e, m}$. The coefficients $A_{n}$ and $B_{n}$ are no longer constants, but become functions of $r$. Although $\Phi^{e, m}$ is still a function of two variables only, $\Phi^{e, m}=\Phi^{e, m}(x, y)$, Eq. (2.5) no longer yields a correct prescription for calculating $\Phi^{e, m}(r, \theta)$ from $\Phi^{e, m}(x, y)$.

\subsection{Equations for Scalar and Vector Potentials with Curved $z$ Axis}

We seek a set of functions which will yield a corresponding multipole expansion when the $z$ axis is curved. In curvilinear coordinates, Laplace's equation for a scalar $V$ does not have the same form as that for a component of a vector $A_{\phi}$. Nevertheless, the twin problems of solving for $V$ and $A_{\phi}$, when the $z$ axis is curved, are closely related, and so we shall treat them together. In cylindrical coordinates $(\rho, \phi, y)$, Laplace's equation has the form

$$
\frac{1}{\rho} \frac{\partial}{\partial \rho}\left(\rho \frac{\partial V}{\partial \rho}\right)+\frac{\partial^{2} V}{\partial y^{2}}+\frac{1}{\rho^{2}} \frac{\partial^{2} V}{\partial \phi^{2}}=0 .
$$

for a scalar $V$. Here the origin is at $\rho=0$, the center of curvature. We define the $x$ axis to be radial, and $z$ to be longitudinal (the $\phi$ direction). Suppose the $z$ axis has a radius of curvature $\rho_{0}=\rho_{0}(z)$, or curvature $h=\rho_{0}^{-1}$. We shift the origin to $\rho=\rho_{0}$, hence $\rho=\rho_{0}+x=\rho_{0}(1+h x)$. Then

$$
d \rho=d x, \quad d z=\rho_{0} d \phi, \quad \rho d \phi=(1+h x) \rho_{0} d \phi=(1+h x) d z,
$$

and so in the "curved $z$ axis coordinate system" Laplace's equation for a scalar $V$ has the form

$$
\frac{1}{1+h x} \frac{\partial}{\partial x}\left((1+h x) \frac{\partial V}{\partial x}\right)+\frac{\partial^{2} V}{\partial y^{2}}+\frac{1}{1+h x} \frac{\partial}{\partial z}\left(\frac{1}{1+h x} \frac{\partial V}{\partial z}\right)=0 .
$$

The above equation is valid even if $h$ is a function of $z$. However, I shall only consider cases where $h$ is constant, and $V=V(x, y)$ only. Then the derivatives in $z$ vanish. Laplace's equation simplifies 
to

$$
\frac{1}{1+h x} \frac{\partial}{\partial x}\left((1+h x) \frac{\partial V}{\partial x}\right)+\frac{\partial^{2} V}{\partial y^{2}}=0
$$

It will be convenient below to use the dimensionless variable $\xi=1+h x$ instead of $x$, in which case the above equation becomes

$$
\frac{h^{2}}{\xi} \frac{\partial}{\partial \xi}\left(\xi \frac{\partial V}{\partial \xi}\right)+\frac{\partial^{2} V}{\partial y^{2}}=0
$$

For the vector potential, Laplace's equation is more complicated. However, if $\vec{A}=A_{\phi} \hat{z}$, and $A_{\phi}$ is a function of $x$ and $y$ only, we obtain the equation

$$
\frac{\partial}{\partial \rho}\left(\frac{1}{\rho} \frac{\partial}{\partial \rho}\left(\rho A_{\phi}\right)\right)+\frac{\partial^{2}}{\partial y^{2}} A_{\phi}=0 .
$$

Shifting the origin, and defining $\xi=1+h x$, etc. yields

$$
\begin{aligned}
0 & =\frac{\partial}{\partial x}\left(\frac{1}{1+h x} \frac{\partial}{\partial x}\left[(1+h x) A_{\phi}\right]\right)+\frac{\partial^{2}}{\partial y^{2}} A_{\phi} \\
& =h^{2} \frac{\partial}{\partial \xi}\left(\frac{1}{\xi} \frac{\partial}{\partial \xi}\left(\xi A_{\phi}\right)\right)+\frac{\partial^{2}}{\partial y^{2}} A_{\phi} .
\end{aligned}
$$

\subsection{General Expressions for Multipoles}

We now need to compile a list of "curved multipoles" analogous to the "straight multipoles" in Eq. (2.3), i.e. curved dipole, skew dipole, quadrupole, skew quadrupole, sextupole, skew sextupole, octupole, etc. for both the scalar and vector potentials. This will provide a set of basis functions (I use the word 'basis' loosely in this context), in terms of which to express the potentials, and eventually the electric and magnetic fields, when the $z$ axis is curved in a circle. To this end, it is useful to note some relationships between multipoles of different order.

Suppose that $\Phi^{e}$ and $\Phi^{m}$ are solutions of Laplace's equation for a scalar and vector potential, respectively, in two dimensions, with a curved $z$ axis, i.e.

$$
\begin{aligned}
\frac{h^{2}}{\xi} \frac{\partial}{\partial \xi}\left(\xi \frac{\partial \Phi^{e}}{\partial \xi}\right)+\frac{\partial^{2} \Phi^{e}}{\partial y^{2}} & =0, \\
h^{2} \frac{\partial}{\partial \xi}\left(\frac{1}{\xi} \frac{\partial}{\partial \xi}\left(\xi \Phi^{m}\right)\right)+\frac{\partial^{2} \Phi^{m}}{\partial y^{2}} & =0 .
\end{aligned}
$$

Let us define $\Psi^{e, m}=\partial \Phi^{e, m} / \partial y$. We see immediately that $\Psi^{e}$ and $\Psi^{m}$ are also solutions of the appropriate forms of Laplace's equation in two dimensions, with a curved $z$ axis, because the partial derivative $\partial / \partial y$ commutes with all the derivatives in Eq. (2.14). For example

$$
\frac{h^{2}}{\xi} \frac{\partial}{\partial \xi}\left(\xi \frac{\partial \Psi^{e}}{\partial \xi}\right)+\frac{\partial^{2} \Psi^{e}}{\partial y^{2}}=\frac{h^{2}}{\xi} \frac{\partial}{\partial \xi}\left(\xi \frac{\partial^{2} \Phi^{e}}{\partial \xi \partial y}\right)+\frac{\partial^{3} \Phi^{e}}{\partial y^{3}}
$$




$$
=\frac{\partial}{\partial y}\left[\frac{h^{2}}{\xi} \frac{\partial}{\partial \xi}\left(\xi \frac{\partial \Phi^{e}}{\partial \xi}\right)+\frac{\partial^{2} \Phi^{e}}{\partial y^{2}}\right]=0 .
$$

We can turn the above result around, within limits to be described below, to deduce that if $\Psi^{e, m}$ is a solution of Laplace's equation (in two dimensions, etc.), then defining

$$
\Upsilon^{e, m} \equiv \int^{y} \Psi^{e, m}\left(x, y^{\prime}\right) d y^{\prime}
$$

will also yield a solution of Laplace's equation. Hence we can obtain higher order multipoles by systematically integrating lower order multipoles with respect to $y$. The catch is that the integrals are indeterminate up to the addition of an arbitrary function of $x$. Hence we can obtain higher order multipoles from lower order multipoles provided we can find a way to determine the terms which depend only on $x$.

The following prescription provides the necessary functions. We leave the $y$ dependence alone in all the expressions for the straight multipoles, in Eqs. (2.3) and (2.4), and generalize the $x$ dependence via $x^{n} \rightarrow U_{n}^{e}(x)$ for the electrostatic scalar potential, and $x^{n} \rightarrow U_{n}^{m}(x)$ for the magnetic vector potential, where $U_{n}^{e}(x)$ and $U_{n}^{m}(x)$ are functions of $x$ only, to be determined below. In other words, we search for functions $U_{n}^{e_{i} m}(x)$ such that the expressions for the first few curved multipoles will be

$$
\begin{array}{lll}
\widetilde{\Phi}_{0}^{n}=U_{0}^{e, m}, & \widetilde{\Phi}_{0}^{s}=0, \\
\widetilde{\Phi}_{1}^{n}=U_{1}^{e, m}, & \widetilde{\Phi}_{1}^{s}=U_{0}^{e, m} y, \\
\widetilde{\Phi}_{2}^{n}=U_{2}^{e, m}-U_{0}^{e, m} y^{2}, & \widetilde{\Phi}_{2}^{s}=2 U_{1}^{e, m} y, \\
\widetilde{\Phi}_{3}^{n}=U_{3}^{e, m}-3 U_{1}^{e, m} y^{2}, & \widetilde{\Phi}_{3}^{s}=3 U_{2}^{e, m} y-U_{0}^{e, m} y^{3}, \\
\widetilde{\Phi}_{4}^{n}=U_{4}^{e, m}-6 U_{2}^{e, m} y^{2}+U_{0}^{e, m} y^{4}, & \widetilde{\Phi}_{4}^{s}=4 U_{3}^{e, m} y-4 U_{1}^{e, m} y^{3},
\end{array}
$$

where we put a tilde on $\Phi$ to denote that the $z$ axis is curved, and the superscripts $n$ and $s$ on $\widetilde{\Phi}^{n, s}$ denote normal and skew multipoles, respectively, and we drop the superscripts $\{e, m\}$ on $\tilde{\Phi}^{e, m}$ to avoid cluttering the notation. We can easily verify that

$$
\frac{\partial \widetilde{\Phi}_{n}^{n}}{\partial y}=-n \widetilde{\Phi}_{n-1}^{s}, \quad \frac{\partial \widetilde{\Phi}_{n}^{s}}{\partial y}=n \widetilde{\Phi}_{n-1}^{n},
$$

i.e. if $\widetilde{\Phi}_{n}^{n, s}$ is a solution of Laplace's equation with a curved $z$ axis, then $\partial \widetilde{\Phi}_{n}^{n, s} / \partial y$ is (proportional to) a multipole of one order lower, and hence is also a solution of Laplace's equation. The general formula for the curved multipoles is given by modifying Eq. (2.4) in the obvious way:

$$
\begin{aligned}
& \widetilde{\Phi}_{n}^{n}=\sum_{k=0}^{K_{1}}(-1)^{k} \frac{n !}{(n-2 k) !(2 k) !} U_{n-2 k}^{e, m} y^{2 k} \\
& \widetilde{\Phi}_{n}^{s}=\sum_{k=0}^{K_{2}}(-1)^{k} \frac{n !}{(n-2 k-1) !(2 k+1) !} U_{n-2 k-1}^{e, m} y^{2 k+1} .
\end{aligned}
$$

We now need to determine the functions $U_{n}^{e, m}$, for $n=0,1,2, \ldots$ 


\subsection{Tabulation of $U_{n}^{e, m}$ Functions}

We shall present the details of the derivations of $U_{n}^{e}$ and $U_{n}^{m}$ later. For ease of reference, and to justify that the required functions do exist, we list the explicit expressions for $U_{n}^{e}$ and $U_{n}^{m}$ for $n=0$ through 6 in closed form below:

$$
\begin{aligned}
U_{0}^{e} & =1, \\
U_{1}^{e} & =h^{-1} \ln \xi \\
U_{2}^{e} & =\frac{1}{2 h^{2}}\left(\xi^{2}-1-2 \ln \xi\right), \\
U_{3}^{e} & =\frac{3}{2 h^{3}}\left(1-\xi^{2}+\left(1+\xi^{2}\right) \ln \xi\right), \\
U_{4}^{e} & =\frac{3}{8 h^{4}}\left(-5+4 \xi^{2}+\xi^{4}-4\left(1+2 \xi^{2}\right) \ln \xi\right), \\
U_{5}^{e} & =\frac{15}{16 h^{5}}\left(3\left(1-\xi^{4}\right)+2\left(1+4 \xi^{2}+\xi^{4}\right) \ln \xi\right), \\
U_{6}^{e} & =\frac{5}{16 h^{6}}\left(-10-9 \xi^{2}+18 \xi^{4}+\xi^{6}-6\left(1+6 \xi^{2}+3 \xi^{4}\right) \ln \xi\right),
\end{aligned}
$$

and

$$
\begin{aligned}
U_{0}^{m} & =\frac{1}{\xi} \\
U_{1}^{m} & =\frac{1}{2 h}\left(\xi-\frac{1}{\xi}\right) \\
U_{2}^{m} & =\frac{1}{2 h^{2}}\left(\frac{1}{\xi}-\xi+2 \xi \ln \xi\right) \\
U_{3}^{m} & =\frac{3}{8 h^{3}}\left(-\frac{1}{\xi}+\xi^{3}-4 \xi \ln \xi\right) \\
U_{4}^{m} & =\frac{3}{8 h^{4}}\left(\frac{1}{\xi}+4 \xi-5 \xi^{3}+4\left(2 \xi+\xi^{3}\right) \ln \xi\right) \\
U_{5}^{m} & =\frac{5}{16 h^{5}}\left(-\frac{1}{\xi}-9 \xi+9 \xi^{3}+\xi^{5}-12\left(\xi+\xi^{3}\right) \ln \xi\right) \\
U_{6}^{m} & =\frac{5}{16 h^{6}}\left(\frac{1}{\xi}+18 \xi-9 \xi^{3}-10 \xi^{5}+6\left(3 \xi+6 \xi^{3}+\xi^{5}\right) \ln \xi\right) .
\end{aligned}
$$

It is also convenient to expand them in power series in $x$, especially when $\xi \simeq 1$, i.e. $x \ll \rho_{0}$ (which will the case in many applications), because the series expansions will converge rapidly, whereas the closed form expressions may involve delicate cancellations when $\xi \simeq 1$. We find

$$
U_{1}^{e}=x-\frac{h x^{2}}{2}+\frac{h^{2} x^{3}}{3}-\frac{h^{3} x^{4}}{4}+\cdots+(-1)^{n} \frac{h^{n} x^{n+1}}{n+1}+\cdots
$$




$$
\begin{aligned}
& U_{2}^{e}=x^{2}-\frac{h x^{3}}{3}+\frac{h^{2} x^{4}}{4}-\frac{h^{3} x^{5}}{5}+\cdots+(-1)^{n} \frac{h^{n} x^{n+2}}{n+2}+\cdots \\
& U_{3}^{e}=x^{3}-\frac{3}{2} \sum_{n=4}^{\infty}(-1)^{n}\left(\frac{2}{n}-\frac{2}{n-1}+\frac{1}{n-2}\right) h^{n-3} x^{n} \\
& U_{4}^{e}=x^{4}+\frac{3}{2} \sum_{n=5}^{\infty}(-1)^{n}\left(\frac{3}{n}-\frac{4}{n-1}+\frac{2}{n-2}\right) h^{n-4} x^{n} \\
& U_{5}^{e}=x^{5}-\frac{15}{8} \sum_{n=6}^{\infty}(-1)^{n}\left(\frac{6}{n}-\frac{12}{n-1}+\frac{10}{n-2}-\frac{4}{n-3}+\frac{1}{n-4}\right) h^{n-5} x^{n}, \\
& U_{6}^{e}=x^{6}+\frac{15}{8} \sum_{n=7}^{\infty}(-1)^{n}\left(\frac{10}{n}-\frac{24}{n-1}+\frac{24}{n-2}-\frac{12}{n-3}+\frac{3}{n-4}\right) h^{n-6} x^{n},
\end{aligned}
$$

and

$$
\begin{aligned}
& U_{0}^{m}=1-h x+h^{2} x^{2}-h^{3} x^{3}+\cdots+(-1)^{n} h^{n} x^{n}+\cdots \\
& U_{1}^{m}=x-\frac{h x^{2}}{2}+\frac{h^{2} x^{3}}{2}-\frac{h^{3} x^{4}}{2}+\cdots+(-1)^{n-1} \frac{h^{n-1} x^{n}}{2}+\cdots \\
& U_{2}^{m}=x^{2}-\frac{2}{3} h x^{3}+\frac{7}{12} h^{2} x^{4}-\frac{11}{20} h^{3} x^{5}+\cdots+(-1)^{n}\left[\frac{1}{2}+\frac{1}{n(n-1)}\right] h^{n-2} x^{n}+\cdots \\
& U_{3}^{m}=x^{3}-\frac{3}{8} \sum_{n=4}^{\infty}(-1)^{n}\left[1+\frac{4}{n(n-1)}\right] h^{n-3} x^{n}, \\
& U_{4}^{m}=x^{4}+\frac{3}{8} \sum_{n=5}^{\infty}(-1)^{n}\left[1-4\left(\frac{3}{n}-\frac{5}{n-1}+\frac{3}{n-2}-\frac{1}{n-3}\right)\right] h^{n-4} x^{n}, \\
& U_{5}^{m}=x^{5}-\frac{5}{16} \sum_{n=6}^{\infty}(-1)^{n}\left[1-12\left(\frac{2}{n}-\frac{4}{n-1}+\frac{3}{n-2}-\frac{1}{n-3}\right)\right] h^{n-5} x^{n}, \\
& U_{6}^{m}=x^{6}+\frac{5}{16} \sum_{n=7}^{\infty}(-1)^{n}\left[1-6\left(\frac{10}{n}-\frac{26}{n-1}+\frac{28}{n-2}-\frac{16}{n-3}\right.\right. \\
& \left.\left.+\frac{5}{n-4}-\frac{1}{n-5}\right)\right] h^{n-6} x^{n} .
\end{aligned}
$$

\subsection{Recursion Relations for $U_{n}^{e, m}$ Functions}

In this section, we derive recursion relations for $U_{n}^{e}$ and $U_{n}^{m}$, and various other useful relationships between these functions. From the general expression for $\widetilde{\Phi}_{n}^{n}$ in Eq. (2.19), we see that

$$
\widetilde{\Phi}_{n}^{n}=U_{n}^{e, m}-\frac{n(n-1)}{2} U_{n-2}^{e, m} y^{2}+\cdots
$$


Let us use $\xi=1+h x$ and put $U_{n}^{e, m}=U_{n}^{e, m}(\xi)$. We begin with the scalar potential, so we treat $U_{n}^{e}$ first. Substitution into Eq. (2.11) yields

$$
\frac{h^{2}}{\xi} \frac{\partial}{\partial \xi}\left(\xi \frac{\partial \tilde{\Phi}_{n}^{n}}{\partial \xi}\right)+\frac{\partial^{2} \widetilde{\Phi}_{n}^{n}}{\partial y^{2}}=0 .
$$

Retaining only terms which do not depend on $y$ after differentiation, we find that

$$
\begin{aligned}
\frac{h^{2}}{\xi} \frac{d}{d \xi}\left(\xi \frac{d U_{n}^{e}}{d \xi}\right)-n(n-1) U_{n-2}^{e} & =0 \\
\frac{1}{\xi} \frac{d}{d \xi}\left(\xi \frac{d U_{n}^{e}}{d \xi}\right) & =\frac{n(n-1)}{h^{2}} U_{n-2}^{e} .
\end{aligned}
$$

This can be solved recursively to determine the $U_{n}^{e}$ 's, provided we supply expressions for $U_{0}^{e}$ and $U_{1}^{e}$. We shall do so later. In any case, we know that, in the limit of zero curvature $h \rightarrow 0, U_{n}^{e} \simeq x^{n}$. First, however, we turn to the vector potential, and the $U_{n}^{m}$ functions. The recursion relation for $U_{n}^{m}$ is easily found to be

$$
\frac{d}{d \xi}\left[\frac{1}{\xi} \frac{d}{d \xi}\left(\xi U_{n}^{m}\right)\right]=\frac{n(n-1)}{h^{2}} U_{n-2}^{m} .
$$

We can now derive a remarkably simple relation between the $U_{n}^{m}$ 's and $U_{n}^{e}$ 's. Differentiating Eq. (2.26) with respect to $\xi$, we deduce that

$$
\frac{d}{d \xi}\left[\frac{1}{\xi} \frac{d}{d \xi}\left(\xi \frac{d U_{n}^{e}}{d \xi}\right)\right]=\frac{n(n-1)}{h^{2}} \frac{d U_{n-2}^{e}}{d \xi} .
$$

This is exactly the same as Eq. (2.27), with $d U_{n}^{e} / d \xi$ in place of $U_{n}^{m}$ ! This is a slightly hasty identification of $U_{n}^{m}$ with $d U_{n}^{e} / d \xi$, however. Note that, in the limit $h \rightarrow 0$, or rather $|h x| \ll 1$, we expect $U_{n}^{e} \simeq U_{n}^{m} \simeq x^{n}$, hence $d U_{n}^{e} / d \xi \simeq n x^{n-1} / h$. The correct relation between $U_{n}^{m}$ and $U_{n}^{e}$ is therefore

$$
\frac{d U_{n}^{e}}{d \xi}=\frac{n}{h} U_{n-1}^{m}
$$

This can be verified to be consistent with Eqs. (2.27) and (2.28). It is therefore unnecessary to derive the $U_{n}^{e}$ 's and $U_{n}^{m}$ 's separately.

The above relation, Eq. (2.29), although simple and elegant, has one disadvantage: to calculate $U_{n}^{m}$, we need to know $U^{e}$ to one order higher, i.e. $U_{n+1}^{e}$. This may involve unnecessary effort, because the labor involved in solving for $U_{n}^{e}$ increases with the value of $n$. It is possible to obtain $U_{n}^{m}$ from $U_{n-1}^{e}$ via the following trick. Using Eqs. (2.26) and (2.29),

$$
\frac{1}{\xi} \frac{d}{d \xi}\left(\xi U_{n-1}^{m}\right)=\frac{h}{n} \frac{1}{\xi} \frac{d}{d \xi}\left(\xi \frac{d U_{n}^{e}}{d \xi}\right)=\frac{n-1}{h} U_{n-2}^{e} .
$$

Multiplying through by $\xi$ and integrating yields

$$
\xi U_{n-1}^{m}=\frac{n-1}{h} \int_{1}^{\xi} \xi^{\prime} U_{n-2}^{e}\left(\xi^{\prime}\right) d \xi^{\prime} .
$$


The lower limit of integration follows from the fact that $U_{n-1}^{m}=0$ at $\xi=1$, for $n \geq 2$. Shifting the index and dividing by $\xi$, we find that

$$
U_{n}^{m}=\frac{n}{h \xi} \int_{1}^{\xi} \xi^{\prime} U_{n-1}^{e}\left(\xi^{\prime}\right) d \xi^{\prime}
$$

which is valid for $n \geq 1$. Hence we can calculate $U_{n}^{e}$ and $U_{n}^{m}$ for $n=0,1,2, \ldots, N$ without having to know $U_{N+1}^{e}$. In fact, we can use Eq. (2.29) to calculate $U_{n}^{e}$ from $U_{n-1}^{m}$ and Eq. (2.32) to calculate $U_{n}^{m}$ from $U_{n-1}^{e}$, as opposed to Eqs. (2.26) and (2.27). Let us do so. Let us write

$$
U_{n-1}^{m}=\frac{a_{-1}}{\xi}+\sum_{k=1}^{\infty} a_{k} \xi^{k}+b_{k} \xi^{k} \ln \xi
$$

The above form for $U_{n-1}^{m}$ will be justified a posteriori in a later section. Then, substituting in Eq. (2.29) yields

$$
\begin{aligned}
\frac{d U_{n}^{e}}{d \xi} & =\frac{n}{h} U_{n-1}^{m}=\frac{n}{h}\left[\frac{a_{-1}}{\xi}+\sum_{k=1}^{\infty} a_{k} \xi^{k}+b_{k} \xi^{k} \ln \xi\right] \\
U_{n}^{e} & =\frac{n}{h}\left[a_{-1} \ln \xi+\sum_{k=1}^{\infty} a_{k} \frac{\xi^{k+1}-1}{k+1}+b_{k}\left(\frac{\xi^{k+1} \ln \xi}{k+1}-\frac{\xi^{k+1}-1}{(k+1)^{2}}\right)\right] .
\end{aligned}
$$

Putting

$$
U_{n}^{e}=\sum_{k=0}^{\infty} c_{k} \xi^{k}+d_{k} \xi^{k} \ln \xi,
$$

the form of which will also be justified a posteriori below, we find that

$$
\begin{aligned}
& c_{0}=\frac{n}{h} \sum_{k=1}^{\infty}\left[-\frac{a_{k}}{k+1}+\frac{b_{k}}{(k+1)^{2}}\right], \\
& c_{k}=\frac{n}{h}\left[\frac{a_{k-1}}{k}-\frac{b_{k-1}}{k^{2}}\right] \quad(k>0), \\
& d_{0}=\frac{n}{h} a_{-1}, \\
& d_{k}=\frac{n}{h} \frac{b_{k-1}}{k} .
\end{aligned}
$$

Furthermore, if we put

$$
U_{n-1}^{e}=\sum_{k=0}^{\infty} c_{k}^{\prime} \xi^{k}+d_{k}^{\prime} \xi^{k} \ln \xi
$$

and substitute into Eq. (2.32), we first obtain

$$
\int_{1}^{\xi} \xi^{\prime} U_{n-1}^{e}\left(\xi^{\prime}\right) d \xi^{\prime}=\sum_{k=0}^{\infty} c_{k}^{\prime} \frac{\xi^{k+2}-1}{k+2}+d_{k}^{\prime}\left[\frac{\xi^{k+2} \ln \xi}{k+2}-\frac{\xi^{k+2}-1}{(k+2)^{2}}\right]
$$


and then

$$
\begin{aligned}
U_{n}^{m}=\frac{n}{h} \int_{1}^{\xi} \xi^{\prime} U_{n-1}^{e}\left(\xi^{\prime}\right) d \xi^{\prime}=\frac{n}{h}\left\{\frac{1}{\xi}\left[\sum_{k=0}^{\infty}-\frac{c_{k}^{\prime}}{k+2}+\frac{d_{k}^{\prime}}{(k+2)^{2}}\right]\right. \\
\left.+\sum_{k=0}^{\infty}\left[\frac{c_{k}^{\prime}}{k+2}-\frac{d_{k}^{\prime}}{(k+2)^{2}}\right] \xi^{k+1}+\frac{d_{k}^{\prime}}{k+2} \xi^{k+1} \ln \xi\right\} .
\end{aligned}
$$

Writing

$$
U_{n}^{m}=\frac{a_{-1}^{\prime}}{\xi}+\sum_{k=1}^{\infty} a_{k}^{\prime} \xi^{k}+b_{k}^{\prime} \xi^{k} \ln \xi
$$

we deduce that

$$
\begin{aligned}
a_{-1}^{\prime} & =\frac{n}{h} \sum_{k=0}^{\infty}\left[-\frac{c_{k}^{\prime}}{k+2}+\frac{d_{k}^{\prime}}{(k+2)^{2}}\right] \\
a_{k}^{\prime} & =\frac{n}{h}\left[\frac{c_{k-1}^{\prime}}{k+1}-\frac{d_{k-1}^{\prime}}{(k+1)^{2}}\right] \quad(k>0) \\
b_{k}^{\prime} & =\frac{n}{h} \frac{d_{k-1}^{\prime}}{k+1} .
\end{aligned}
$$

Eqs. (2.36) and (2.41) can easily be coded into a computer program to yield the coefficients of the terms in $U_{n}^{e}$ and $U_{n}^{m}$ up to arbitrary values of $n$.

It is also useful to express the $U_{n}^{e}$ and $U_{n}^{m}$ as power series in $x$. Although the power series will contain arbitrarily high powers of $x$, i.e. infinitely many terms, as opposed to the closed form expressions, in many, if not most, applications, $|x| \ll \rho_{0}$, or equivalently $|h x| \ll 1$, so the series expansions will converge rapidly. We put

$$
U_{n-1}^{m}=\sum_{k=n-1}^{\infty} \alpha_{k} x^{k}, \quad U_{n}^{e}=\sum_{k=n}^{\infty} \beta_{k} x^{k},
$$

whence, using Eq. (2.29),

$$
\begin{aligned}
\frac{d U_{n}^{e}}{d x} & =n U_{n-1}^{m}, \\
\sum_{k=n}^{\infty} \beta_{k} k x^{k-1} & =n \sum_{k=n-1}^{\infty} \alpha_{k} x^{k},
\end{aligned}
$$

and so we deduce

$$
\beta_{k}=\frac{n}{k} \alpha_{k-1}
$$

Note that $\beta_{n}=\alpha_{n-1}=1$ because $U_{n}^{e} \simeq x^{n}$ and $U_{n-1}^{m} \simeq x^{n-1}$ for $|h x| \rightarrow 0$. Next let us write

$$
U_{n-1}^{e}=\sum_{k=n-1}^{\infty} \beta_{k}^{\prime} x^{k}, \quad U_{n}^{m}=\sum_{k=n}^{\infty} \alpha_{k}^{\prime} x^{k},
$$


and substitute into Eq. (2.32), which yields

$$
\begin{aligned}
U_{n}^{m} & =\frac{n}{1+h x} \int_{0}^{x}\left(1+h x^{\prime}\right) U_{n-1}^{e}\left(x^{\prime}\right) d x^{\prime}, \\
(1+h x) \sum_{k=n}^{\infty} \alpha_{k}^{\prime} x^{k} & =n \int_{0}^{x}\left(1+h x^{\prime}\right) \sum_{k=n-1}^{\infty} \beta_{k}^{\prime} x^{\prime k} d x^{\prime} \\
& =n \sum_{k=n-1}^{\infty} \beta_{k}^{\prime}\left[\frac{x^{k+1}}{k+1}+\frac{h x^{k+2}}{k+2}\right] .
\end{aligned}
$$

Equating powers of $x$, we find

$$
\alpha_{k}^{\prime}+h \alpha_{k-1}^{\prime}=\frac{n}{k}\left(\beta_{k-1}^{\prime}+h \beta_{k-2}^{\prime}\right),
$$

which we rewrite in the form

$$
\begin{aligned}
& \alpha_{n}^{\prime}=\beta_{n-1}^{\prime}=1, \\
& \alpha_{k}^{\prime}=\frac{n}{k}\left(\beta_{k-1}^{\prime}+h \beta_{k-2}^{\prime}\right)-h \alpha_{k-1}^{\prime} \quad(k \geq n+1),
\end{aligned}
$$

where the first line again follows because because $U_{n}^{m} \simeq x^{n}$ and $U_{n-1}^{e} \simeq x^{n-1}$ for $|h x| \rightarrow 0$.

\subsection{Multipoles for Electric and Magnetic Fields with Curved $z$ Axis}

The above expressions in Eqs. (2.17) or (2.19) pertain to the potentials. We also want multipoles for the electric and magnetic fields, i.e. for the components $E_{x}, E_{y}, B_{x}$ and $B_{y}$. When the $z$ axis is straight, this is a trivial problem, because the required functions are the same as for the potentials, viz. Fourier harmonics. The result is slightly more complicated when the $z$ axis is curved.

It can easily be shown that, if $E_{x}, E_{y}, B_{x}$ and $B_{y}$ are functions of $x$ and $y$ only, then they satisfy the equations

$$
\begin{aligned}
\frac{\partial}{\partial r}\left(\frac{1}{r} \frac{\partial}{\partial r}\left(r E_{x}\right)\right)+\frac{\partial^{2} E_{x}}{\partial y^{2}} & =0, \\
\frac{1}{r} \frac{\partial}{\partial r}\left(r \frac{\partial E_{y}}{\partial r}\right)+\frac{\partial^{2} E_{y}}{\partial y^{2}} & =0, \\
\frac{\partial}{\partial r}\left(\frac{1}{r} \frac{\partial}{\partial r}\left(r B_{x}\right)\right)+\frac{\partial^{2} B_{x}}{\partial y^{2}} & =0, \\
\frac{1}{r} \frac{\partial}{\partial r}\left(r \frac{\partial B_{y}}{\partial r}\right)+\frac{\partial^{2} B_{y}}{\partial y^{2}} & =0 .
\end{aligned}
$$


Hence $E_{x}$ and $B_{x}$ satisfy the same equation as $A_{\phi}$, and $E_{y}$ and $B_{y}$ satisfy the same equation as $V$. Hence, to expand $E_{x}$ and $B_{x}$ in multipoles, we use the $U_{n}^{m}$ functions, while to expand $E_{y}$ and $B_{y}$ in multipoles, we use the $U_{n}^{e}$ functions. Explicitly,

$$
\begin{array}{ll}
\tilde{F}_{x 0}^{n}=U_{0}^{m}, & \tilde{F}_{x 0}^{s}=0, \\
\tilde{F}_{x 1}^{n}=U_{1}^{m}, & \tilde{F}_{x 1}^{s}=U_{0}^{m} y, \\
\tilde{F}_{x 2}^{n}=U_{2}^{m}-U_{0}^{m} y^{2}, & \tilde{F}_{x 2}^{s}=2 U_{1}^{m} y, \\
\tilde{F}_{x 3}^{n}=U_{3}^{m}-3 U_{1}^{m} y^{2}, & \tilde{F}_{x 3}^{s}=3 U_{2}^{m} y-U_{0}^{m} y^{3} \\
\tilde{F}_{x 4}^{n}=U_{4}^{m}-6 U_{2}^{m} y^{2}+U_{0}^{m} y^{4}, & \tilde{F}_{x 4}^{s}=4 U_{3}^{m} y-4 U_{1}^{m} y^{3}
\end{array}
$$

etc., for the horizontal components of the fields, and

$$
\begin{array}{ll}
\tilde{F}_{y 0}^{n}=U_{0}^{e}, & \tilde{F}_{y 0}^{s}=0, \\
\tilde{F}_{y 1}^{n}=U_{1}^{e}, & \tilde{F}_{y 1}^{s}=U_{0}^{e} y, \\
\tilde{F}_{y 2}^{n}=U_{2}^{e}-U_{0}^{e} y^{2}, & \tilde{F}_{y 2}^{s}=2 U_{1}^{e} y, \\
\tilde{F}_{y 3}^{n}=U_{3}^{e}-3 U_{1}^{e} y^{2}, & \tilde{F}_{y 3}^{s}=3 U_{2}^{e} y-U_{0}^{e} y^{3}, \\
\tilde{F}_{y 4}^{n}=U_{4}^{e}-6 U_{2}^{e} y^{2}+U_{0}^{e} y^{4}, & \tilde{F}_{y 4}^{s}=4 U_{3}^{e} y-4 U_{1}^{e} y^{3},
\end{array}
$$

etc. for the vertical components of the fields, and so on for higher order multipoles. Here the symbol $F$ denotes either $E$ or $B$, and the tilde indicates that the multipole is for use when the $z$ axis is curved.

\subsection{Explicit Expressions for $U_{n}^{e, m}$ Functions}

We have now built up a fairly elaborate scheme, but still have not yet justified the explicit expressions for the $U_{n}^{e}$ and $U_{n}^{m}$ functions presented above. We now do so. We first note that, in the limit of zero curvature $h \rightarrow 0, U_{n}^{e} \simeq U_{n}^{m} \simeq x^{n}$. We start with $U_{0}^{e}$, for which the equation is

$$
\frac{1}{\xi} \frac{d}{d \xi}\left(\xi \frac{d U_{0}^{e}}{d \xi}\right)=0
$$

We see immediately that a suitable solution is

$$
U_{0}^{e}=1,
$$

which trivially reduces to $U_{0}^{e} \rightarrow x^{0}=1$ as $h \rightarrow 0$. Next, we have

$$
\frac{1}{\xi} \frac{d}{d \xi}\left(\xi \frac{d U_{1}^{e}}{d \xi}\right)=0
$$

also. A suitable solution in this case is

$$
U_{1}^{e}=h^{-1} \ln \xi=h^{-1} \ln (1+h x)
$$




$$
=x-\frac{h x^{2}}{2}+\frac{h^{2} x^{3}}{3}-\frac{h^{3} x^{4}}{4}+\cdots+(-1)^{n} \frac{h^{n} x^{n+1}}{n+1}+\cdots
$$

which clearly reduces, as expected, to $U_{1}^{e} \simeq x^{1}=x$ as $h \rightarrow 0$. Using Eq. (2.29), we find that

$$
U_{0}^{m}=h \frac{d U_{1}^{e}}{d \xi}=\frac{1}{\xi} .
$$

Furthermore, using Eq. (2.32), with $U_{0}^{e}=1$,

$$
\begin{aligned}
U_{1}^{m} & =\frac{1}{h \xi} \int_{1}^{\xi} \xi^{\prime} d \xi^{\prime}=\frac{1}{2 h}\left(\xi-\frac{1}{\xi}\right) \\
& =x-\frac{h x^{2}}{2}+\frac{h^{2} x^{3}}{2}-\frac{h^{3} x^{4}}{2}+\cdots+(-1)^{n} \frac{h^{n} x^{n+1}}{2}+\cdots
\end{aligned}
$$

The next function is $U_{2}^{e}$, for which Eq. (2.29) tells us

$$
\begin{aligned}
\frac{d U_{2}^{e}}{d \xi} & =\frac{2}{h} U_{1}^{m}=\frac{1}{h^{2}}\left(\xi-\frac{1}{\xi}\right) \\
U_{2}^{e} & =\frac{1}{2 h^{2}}\left(\xi^{2}-1-2 \ln \xi\right) \\
& =x^{2}-\frac{h x^{3}}{3}+\frac{h^{2} x^{4}}{4}-\frac{h^{3} x^{5}}{5}+\cdots+(-1)^{n} \frac{h^{n} x^{n+2}}{n+2}+\cdots
\end{aligned}
$$

Using Eq. (2.32), we find that

$$
\begin{aligned}
U_{2}^{m} & =\frac{2}{h \xi} \int_{1}^{\xi} \xi^{\prime} U_{1}^{e} d \xi^{\prime}=\frac{2}{h^{2} \xi} \int_{1}^{\xi} \xi^{\prime} \ln \xi^{\prime} d \xi^{\prime}=\frac{1}{h^{2} \xi}\left(\xi^{2} \ln \xi-\frac{\xi^{2}}{2}+\frac{1}{2}\right) \\
& =\frac{1}{2 h^{2}}\left(\frac{1}{\xi}-\xi+2 \xi \ln \xi\right) \\
& =x^{2}-\frac{2}{3} h x^{3}+\frac{7}{12} h^{2} x^{4}-\frac{11}{20} h^{3} x^{5}+\cdots+(-1)^{n}\left[\frac{1}{2}+\frac{1}{n(n-1)}\right] h^{n-2} x^{n}+\cdots
\end{aligned}
$$

We can continue the integrations using Eqs. (2.29) and (2.32), but it is easier eventually to use Eqs. (2.36) and (2.41), hence let us do so now. Then Eq. (2.36) tells us that

$$
\begin{aligned}
U_{3}^{e} & =\frac{3}{2 h^{3}}\left[\frac{1}{2}+\frac{2}{4}+\left(-\frac{1}{2}-\frac{2}{4}\right) \xi^{2}+\ln \xi+\frac{2}{2} \xi^{2} \ln \xi\right] \\
& =\frac{3}{2 h^{3}}\left(1-\xi^{2}+\left(1+\xi^{2}\right) \ln \xi\right) \\
& =x^{3}-\frac{h x^{4}}{2}+\frac{7}{20} h^{2} x^{5}-\frac{11}{40} h^{3} x^{6}+\cdots \\
& =x^{3}-\frac{3}{2} \sum_{n=4}^{\infty}(-1)^{n}\left[\frac{2}{n}-\frac{2}{n-1}+\frac{1}{n-2}\right] h^{n-3} x^{n} .
\end{aligned}
$$


The expression for $U_{3}^{m}$ is, via Eq. (2.41),

$$
\begin{aligned}
U_{3}^{m} & =\frac{3}{2 h^{3} \xi}\left[\left(\frac{1}{2}-\frac{2}{4}-\frac{1}{4}\right) \frac{1}{\xi}+\left(-\frac{1}{2}+\frac{2}{4}\right) \xi+\frac{\xi^{3}}{4}-\frac{2}{2} \xi \ln \xi\right] \\
& =\frac{3}{8 h^{3}}\left(-\frac{1}{\xi}+\xi^{3}-4 \xi \ln \xi\right) \\
& =x^{3}-\frac{3}{8} \sum_{n=4}^{\infty}(-1)^{n}\left[1+\frac{4}{n(n-1)}\right] h^{n-3} x^{n} .
\end{aligned}
$$

At the next order, the expression for $U_{4}^{e}$ is given by

$$
\begin{aligned}
U_{4}^{e} & =\frac{3}{2 h^{4}}\left[0-\frac{4}{4}-\frac{1}{4}+\left(0+\frac{4}{4}\right) \xi^{2}+\frac{\xi^{4}}{4}-\ln \xi-\frac{4}{2} \xi^{2} \ln \xi\right] \\
& =\frac{3}{8 h^{4}}\left(-5+4 \xi^{2}+\xi^{4}-4\left(1+2 \xi^{2}\right) \ln \xi\right) \\
& =x^{4}+\frac{3}{2} \sum_{n=5}^{\infty}(-1)^{n}\left[\frac{3}{n}-\frac{4}{n-1}+\frac{2}{n-2}\right] h^{n-4} x^{n}
\end{aligned}
$$

As for $U_{4}^{m}$, we have

$$
\begin{aligned}
U_{4}^{m} & =\frac{6}{h^{4} \xi}\left[\left(-\frac{1}{2}+\frac{1}{4}+\frac{1}{4}+\frac{1}{16}\right) \frac{1}{\xi}+\left(\frac{1}{2}-\frac{1}{4}\right) \xi+\left(-\frac{1}{4}-\frac{1}{16}\right) \xi^{3}+\frac{1}{2} \xi \ln \xi+\frac{1}{4} \xi^{3} \ln \xi\right] \\
& =\frac{3}{8 h^{4}}\left[\frac{1}{\xi}+4 \xi-5 \xi^{3}+4\left(2 \xi+\xi^{3}\right) \ln \xi\right] \\
& =x^{4}-\frac{3}{2} \sum_{n=5}^{\infty}(-1)^{n}\left[\frac{3}{n}-\frac{5}{n-1}+\frac{3}{n-2}-\frac{1}{n-3}\right] h^{n-4} x^{n}
\end{aligned}
$$

At the next order, we have

$$
\begin{aligned}
U_{5}^{e} & =\frac{15}{8 h^{5}}\left[-\frac{4}{2}+\frac{8}{4}+\frac{5}{4}+\frac{4}{16}+\left(\frac{4}{2}-\frac{8}{4}\right) \xi^{2}+\left(-\frac{5}{4}-\frac{4}{16}\right) \xi^{4}+\ln \xi+\frac{8}{2} \xi^{2} \ln \xi+\frac{4}{4} \xi^{4} \ln \xi\right] \\
& =\frac{15}{16 h^{5}}\left[3\left(1-\xi^{4}\right)+2\left(1+4 \xi^{2}+\xi^{4}\right) \ln \xi\right] \\
& =x^{5}-\frac{15}{8} \sum_{n=6}^{\infty}(-1)^{n}\left[\frac{6}{n}-\frac{12}{n-1}+\frac{10}{n-2}-\frac{4}{n-3}+\frac{1}{n-4}\right] h^{n-5} x^{n}
\end{aligned}
$$

and

$$
\begin{aligned}
U_{5}^{m}=\frac{15}{8 h^{5} \xi}\left[\left(\frac{5}{2}-\frac{4}{4}\right.\right. & \left.-\frac{4}{4}-\frac{8}{16}-\frac{1}{6}\right) \frac{1}{6}+\left(-\frac{5}{2}+\frac{4}{4}\right) \xi \\
& \left.+\left(\frac{4}{4}+\frac{8}{16}\right) \xi^{3}+\frac{\xi^{5}}{6}-\frac{4}{2} \xi \ln \xi-\frac{8}{4} \xi^{3} \ln \xi\right]
\end{aligned}
$$




$$
\begin{aligned}
& =\frac{5}{16 h^{5}}\left[-\frac{1}{\xi}-9 \xi+9 \xi^{3}+\xi^{5}-12\left(\xi+\xi^{3}\right) \ln \xi\right] \\
& =x^{5}+\frac{15}{4} \sum_{n=6}^{\infty}(-1)^{n}\left[\frac{2}{n}-\frac{4}{n-1}+\frac{3}{n-2}-\frac{1}{n-3}\right] h^{n-5} x^{n} .
\end{aligned}
$$

Next,

$$
\begin{aligned}
U_{6}^{e}= & \frac{15}{8 h^{6}}\left[\frac{9}{2}-\frac{9}{4}-\frac{1}{6}-\frac{12}{4}-\frac{12}{16}+\left(-\frac{9}{2}+\frac{12}{4}\right) \xi^{2}\right. \\
& \left.\quad+\left(\frac{9}{4}+\frac{12}{16}\right) \xi^{4}+\frac{\xi^{6}}{6}-\frac{12}{2} \xi^{2} \ln \xi-\frac{12}{4} \xi^{4} \ln \xi\right] \\
= & \frac{5}{16 h^{6}}\left[-10-9 \xi^{2}+18 \xi^{4}+\xi^{6}-6\left(1+6 \xi^{2}+3 \xi^{4}\right) \ln \xi\right] \\
= & x^{6}+\frac{15}{8} \sum_{n=7}^{\infty}(-1)^{n}\left(\frac{10}{n}-\frac{24}{n-1}+\frac{24}{n-2}-\frac{12}{n-3}+\frac{3}{n-4}\right) h^{n-6} x^{n},
\end{aligned}
$$

and

$$
\begin{aligned}
U_{6}^{m}= & \frac{45}{8 h^{6} \xi}\left[\left(-\frac{3}{2}+\frac{0}{4}+\frac{3}{6}+\frac{2}{4}+\frac{8}{16}+\frac{2}{36}\right) \frac{1}{\xi}+\left(\frac{3}{2}-\frac{2}{4}\right) \xi+\left(-\frac{0}{4}-\frac{8}{16}\right) \xi^{3}\right. \\
& \left.\quad+\left(-\frac{3}{6}-\frac{2}{36}\right) \xi^{5}+\frac{2}{2} \xi \ln \xi+\frac{8}{4} \xi^{3} \ln \xi+\frac{2}{6} \xi^{5} \ln \xi\right] \\
= & \frac{45}{8 h^{6}}\left[\frac{1}{18 \xi}+\xi-\frac{\xi^{3}}{2}-\frac{5 \xi^{5}}{9}+\left(\xi+2 \xi^{3}+\frac{\xi^{5}}{3}\right) \ln \xi\right] \\
= & \frac{5}{16 h^{6}}\left[\frac{1}{\xi}+18 \xi-9 \xi^{2}-10 \xi^{5}+6\left(3 \xi+6 \xi^{3}+\xi^{5}\right) \ln \xi\right] \\
= & x^{6}+\frac{5}{16} \sum_{n=7}^{\infty}(-1)^{n}\left[1-6\left(\frac{10}{n}-\frac{26}{n-1}+\frac{28}{n-2}-\frac{16}{n-3}+\frac{5}{n-4}-\frac{1}{n-5}\right)\right] .
\end{aligned}
$$

We can continue this process to any desired order. It is simpler to automate the process by coding it into a computer program, rather than to write out the expressions explicitly.

\subsection{Fitting of Boundary Conditions}

\subsubsection{General Procedure}

We now have a set of 'basis' functions in terms of which to express the potentials $V$ and $A_{\phi}$ and the fields $E_{x}$, etc. To determine the potential and fields for a given system, we need a procedure to fit the boundary conditions for that system. Such a procedure is of particular interest for the fitting 
of analytical functions, i.e. $\widetilde{\Phi}_{n}^{n, s}$, to experimental measurements of magnetic fields taken using Hall probes, etc. However, it is simpler, for pedagogical purposes, to treat examples where we fit only the electrostatic potential to specified boundary conditions. This means that we need treat only the $U_{n}^{e}$ functions. Fitting the electric or magnetic field, or the magnetic vector potential, involves basically the same procedure: we merely use $U_{n}^{m}$ instead of $U_{n}^{e}$, whenever appropriate.

For simplicity, I shall treat only Dirichlet boundary conditions, i.e. I shall assume the value of $V$ is given on some surface which bounds the region where we wish to calculate $V$. Otherwise, if we are given the derivative of $V$, i.e. the electric field, we may need to use a mixture of $U_{n}^{e}$ and $U_{n}^{m}$ functions. When the $z$ axis is straight, one can express an arbitrary potential, in a region that does not enclose any charges, as a sum of straight multipoles, using the Fourier series expansion in Eq. (2.6). When the $z$ axis is curved, we express the potential as a sum of curved multipoles (in a region that encloses no charges) via

$$
V=\tilde{B}_{0}+\sum_{n=1}^{\infty} \tilde{B}_{n} \tilde{V}_{n}^{n}+\tilde{A}_{n} \tilde{V}_{n}^{s},
$$

where the $\widetilde{A}_{n}$ and $\widetilde{B}_{n}$ are constants, and have units of Volts $/(\text { meter })^{n}$, just like the $A_{n}$ and $B_{n}$ in Eq. (2.6). I have included an additive constant $\widetilde{B}_{0}$ as well, because $\widetilde{B}_{0}=V(0,0)$, the value of the potential at the origin, which is not zero in general.

The coefficients $\tilde{A}_{n}$ and $\tilde{B}_{n}$ are determined as follows. The boundary conditions tell us the value of $V$ on some surface which bounds the region where we wish to calculate $V$. We select a set of points $\left(x_{i}, y_{i}\right), i=1,2, \ldots N$ on this bounding surface, and obtain a set of simulaneous equations

$$
\tilde{B}_{0}+\sum_{n=1}^{\infty} \tilde{B}_{n} \tilde{V}_{n}^{n}\left(x_{i}, y_{i}\right)+\tilde{A}_{n} \tilde{V}_{n}^{s}\left(x_{i}, y_{i}\right)=V\left(x_{i}, y_{i}\right),
$$

for $i=1,2, \ldots N$. In the above equation, the unknowns are $\tilde{A}_{n}$ and $\tilde{B}_{n}$. The value of $V\left(x_{i}, y_{i}\right)$ is known. The total set of $N$ equations is solved for $\widetilde{A}_{n}$ and $\widetilde{B}_{n}$. In the special case of an expansion in orthogonal functions, we can let $N \rightarrow \infty$, and fit an infinite set of coefficients, and the sums become integrals - this is implicitly the procedure one uses to determine the $a_{n}$ and $b_{n}$ coefficients of the $r^{n} \sin (n \theta)$ and $r^{n} \cos (n \theta)$ multipoles in Eq. (2.5). The "bounding surface" is a circle of radius $r_{n o r m}$. Unfortunately, the curved multipoles $\widetilde{V}_{n}^{n, s}$ in Eq. (2.68) are not orthogonal (or, strictly speaking, I have not found a suitable weight function with respect to which they are orthogonal). Hence the solution of Eq. (2.69) is more complicated. What one does, therefore, is to use only a finite number of multipoles in the sum in Eq. (2.69). We choose $N$ to be a large but finite number, and perform a least-squares fit to determine the $\widetilde{A}_{n}$ and $\widetilde{B}_{n}$. Note that the value of $N$ must equal or exceed the number of coefficients being fitted. Note also that, in general, this procedure yields only approximate values for $\widetilde{A}_{n}$ and $\widetilde{B}_{n}$. We can illustrate the above ideas by treating some model examples below.

First, however, let us digress briefly to discuss the important problem of fitting a set of multipoles to experimental magnetic field measurements. Typically, for a dipole magnet, one measures the vertical component of the magnetic field in the magnet midplane, i.e. one obtains a set of values for $B_{y}$ as a function of $x$. Far away from the ends of a magnet, the field does not depend on $z$. 
The structure of Laplace's equation guarantees that, if $B_{y}$ is independent of $z$, a knowledge of $B_{y}=B_{y}(x, y=0)$ is sufficient to determine $B_{y}(x, y)$ completely. Hence one expresses $B_{y}(x, y)$ as a polynomial in $x$ and $y$, which satisfies Laplace's equation, and fits the coefficients of the polynomial to the experimental data (for which $y=0$ ), using a least-squares fit. To express the field using curved multipoles, we simply expand $B_{y}$ as a sum of curved multipoles, and this time the least-squares fit gives the coefficients of the curved multipoles. There is no "bounding surface" in this case, instead we fit to data in the magnet midplane. Otherwise there is no difference to the procedure described above for calculating the electrostatic potential $V$ using Dirichlet boundary conditions.

\subsubsection{Dipole}

The first example I shall treat is a horizontal dipole. The system consists of two vertical cylindrical plates which extend to $\pm \infty$ in the vertical direction, as sketched in Fig. 1. The inner plate is at $\rho=\rho_{0}-a$, or $x=-a$, and the outer one is at $\rho=\rho_{0}+a$, or $x=a$, since $x=0$ is at $\rho=\rho_{0}$. The boundary conditions are $V=-V_{0}$ at $x=-a$ and $V=V_{0}$ at $x=a$.

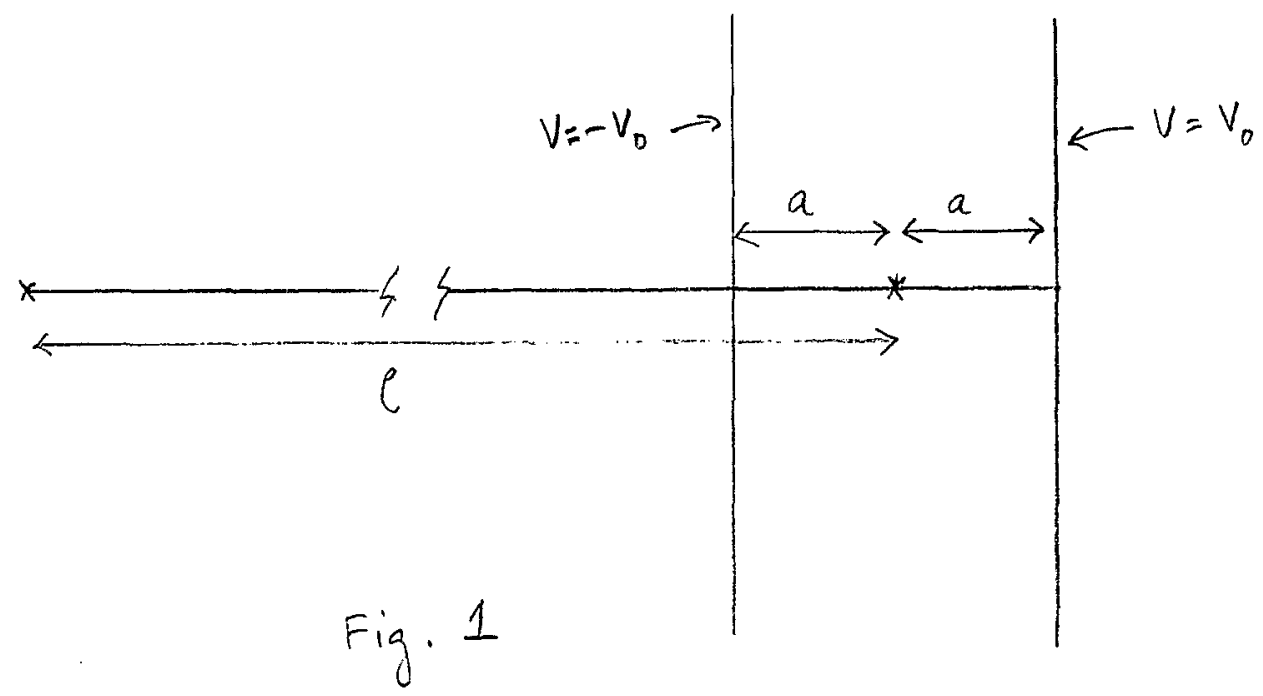

It is good to study this system first because we know that the solution is exactly expressible using only two curved multipoles, viz. the curved dipole term $\tilde{V}_{1}^{n}$ plus a constant $\tilde{V}_{0}^{n}$, i.e.

$$
V=A+B \ln (1+h x)=A+B \ln \xi=A \tilde{V}_{0}^{n}+B h \tilde{V}_{1}^{n},
$$

where $A$ and $B$ are constants, not related to the $A_{n}$ and $B_{n}$ above. Hence we can check the accuracy of the least-squares fitting procedure in Eq. (2.68) because we know the exact answer. It is relatively simple to show that the exact solution for the potential is

$$
V=2 V_{0} \ln \left(\frac{1+h x}{\sqrt{1-h^{2} a^{2}}}\right) / \ln \left(\frac{1+h a}{1-h a}\right),
$$


i.e.

$$
A=V_{0} \frac{\ln (1-h a)+\ln (1+h a)}{\ln (1-h a)-\ln (1+h a)}, \quad B=\frac{2 V_{0}}{\ln (1+h a)-\ln (1-h a)} .
$$

We use eleven terms in Eq. (2.68), i.e. we put

$$
V=\tilde{B}_{0}+\sum_{n=1}^{5} \tilde{B}_{n} \tilde{V}_{n}^{n}+\tilde{A}_{n} \tilde{V}_{n}^{s}
$$

Let us put $a=5 \mathrm{~cm}, \rho_{0}=1000 \mathrm{~cm}$, and $V_{0}=10^{4}$ Volts. For the points $\left(x_{i}, y_{i}\right)$, we fit to 21 points on each plate, so $N=42$ and we put $y_{i}=(i-11) / 2 \mathrm{~cm}$ at $x=-a$, for $i=1, \ldots 21$ and $y_{i}=(i-32) / 2 \mathrm{~cm}$ at $x=a$, for $i=22, \ldots 42$, so $-5 \leq y \leq 5 \mathrm{~cm}$ on each plate. The resulting values of the coefficients are shown in Table 1 . We find that only the constant and dipole terms, viz. $\widetilde{B}_{0}$ and $\widetilde{B}_{1}$, are significantly nonzero, as expected. Further, one can easily verify that the values of $\widetilde{B}_{0}(=A)$ and $\widetilde{B}_{1}(=B h=B / \rho)$ agree with the exact analytical values determined from Eq. (2.72), using the numerical values for $V_{0}, a$ and $\rho_{0}$ quoted above.

Table 1

\begin{tabular}{lrr}
\hline M.ultipole & Normal & Skew \\
\hline 0 & $0.3515 \mathrm{E}+01$ & $0.0000 \mathrm{E}+00$ \\
1 & $0.2000 \mathrm{E}+03$ & $0.5283 \mathrm{E}-30$ \\
2 & $0.6487 \mathrm{E}-31$ & $-0.2265 \mathrm{E}-32$ \\
3 & $-0.2892 \mathrm{E}-32$ & $-0.8384 \mathrm{E}-32$ \\
4 & $-0.4777 \mathrm{E}-33$ & $0.9718 \mathrm{E}-34$ \\
5 & $0.3478 \mathrm{E}-34$ & $0.3181 \mathrm{E}-34$ \\
\hline
\end{tabular}

\subsubsection{Torus with $\cos (2 \theta)$ Potential}

Let us now consider a more complicated system, but where we still know the exact answer when the $z$ axis is straight. Consider a cylinder of radius $a$, and suppose the potential on the surface of the cylinder is given by $V=V_{0} \cos (2 \theta)$, where $x=r \cos \theta$ and $y=r \sin \theta$ and $r=a$ on the surface of the cylinder. Then we know that, if the longitudinal or $z$ axis of the cylinder is straight, the potential inside the cylinder is an exact quadrupole:

$$
V=\frac{V_{0}}{a^{2}} r^{2} \cos (2 \theta) \text {. }
$$

Now suppose that the $z$ axis is bent in a circle of radius $\rho_{0}$, so that the cylinder forms a torus, but the potential on the surface is still given by $V_{0} \cos (2 \theta)$, as sketched in Fig. 2. 
We do not know the exact solution for the potential in this case, but we expect that it will be dominated by the curved quadrupole term $\tilde{V}_{2}^{n}$. We again put $a=5 \mathrm{~cm}, \rho_{0}=1000 \mathrm{~cm}$, and $V_{0}=10^{1}$ Volts. This time we use twenty-three terms in Eq. (2.68), i.e. we put

$$
V=\widetilde{B}_{0}+\sum_{n=1}^{11} \widetilde{B}_{n} \bar{V}_{n}^{n}+\tilde{A}_{n} \tilde{V}_{n}^{s} .
$$

We fit to 100 points spaced equally around the circumference of the cylinder, in the $(x, y)$ plane. The resulting values of the coefficients are shown in Table 2. As expected, we find that the largest term is the quadrupole. In fact, the coefficient of the quadrupole is exactly $V_{0} / a^{2}=10^{4} / 25=400$, which shows that the effects of curvature are small in this case. There are also distinctly nonzero constant and dipole terms because the curvature of the $z$ axis shifts the origin of the quadrupole away from the center of the cylinder, and a sextupole term because of the curvature of the $z$ axis. It is encouraging that the coefficients of the higher order multipoles rapidly become very small, to the extent of being negligible. This shows that we have used an adequate number of multipoles in the sum in Eq. (2.75). As expected, the coefficients of the skew multipoles are very small.

Table 2

\begin{tabular}{lrr}
\hline Multipole & Normal & Skew \\
\hline 0 & $-7.8126 \mathrm{E}+01$ & $0.0000 \mathrm{E}+00$ \\
1 & 2.5000 & $1.0588 \mathrm{E}-10$ \\
2 & 400.0000 & $-2.7707 \mathrm{E}-13$ \\
3 & $3.3334 \mathrm{E}-02$ & $-2.0544 \mathrm{E}-12$ \\
4 & $-4.1667 \mathrm{E}-06$ & $-5.7360 \mathrm{E}-13$ \\
5 & $1.5626 \mathrm{E}-09$ & $-4.3726 \mathrm{E}-14$ \\
6 & $-3.9437 \mathrm{E}-13$ & $1.5158 \mathrm{E}-14$ \\
7 & $-2.6099 \mathrm{E}-16$ & $-1.0106 \mathrm{E}-15$ \\
8 & $4.0104 \mathrm{E}-20$ & $-8.0443 \mathrm{E}-17$ \\
9 & $-2.0860 \mathrm{E}-22$ & $9.0808 \mathrm{E}-21$ \\
10 & $-2.9924 \mathrm{E}-25$ & $-6.7981 \mathrm{E}-25$ \\
11 & $5.0542 \mathrm{E}-29$ & $-1.0268 \mathrm{E}-26$ \\
\hline
\end{tabular}

We cannot compare to an exact solution, so we must use some other method to determine the goodness of the least-squares fit. Note that, since we have used only a finite sum of curved multipoles, and we have fitted to only a finite number of points, the value of $V$ in Eq. (2.75), on the surface of the cylinder, will not be exactly $V_{0} \cos (2 \theta)$. We can plot the difference, say $V_{b c}-V_{l s}$ against $\theta$, where " $b c$ " means "boundary condition" and " $l s "$ means "least-squares." The resulting 
graph is shown in Fig. 3. We see that the difference between $V_{b c}$ and $V_{l s}$ is of $O\left(10^{-13}\right)$ Volts. Recalling that $\left|V_{b c}\right|=O\left(10^{4}\right)$ Volts, this is an excellent fit to the boundary conditions.

In the above calculation, we put $a=5 \mathrm{~cm}$ and $\rho_{0}=1000 \mathrm{~cm}$, which means that $a / \rho_{0}=0.005$, so the effects of the curvature of the $z$ axis were not in fact very large. Let us deliberately increase the curvature effects by repeating the calculation with $\rho_{0}=50 \mathrm{~cm}$, and $a=5 \mathrm{~cm}$ and $V_{0}=10^{4}$ Volts as before. We again use twenty-three terms, as in Eq. (2.75). The values of the coefficients of the multipoles are shown in Table 3. This time the coefficient of the quadrupole is 399.8327 which is slightly different from $V_{0} / a^{2}=400$, which shows that the curvature of the $z$ axis has indeed modified the quadrupole coefficient. We again investigate the goodness of the least-squares fit by plotting $V_{b c}-V_{l s}$ against $\theta$, in Fig. 4. We find that $\left|V_{b c}-V_{l s}\right|=O\left(10^{-12}\right)$ Volts for $\left|V_{b c}\right|=O\left(10^{4}\right)$ Volts, which is again a good fit. Hence we see that, even when the effects of curvature are of order $a / \rho_{0}=5 / 50=0.1$, the use of the curved multipoles and the least-squares fitting procedure yields a good fit to the boundary conditions.

Table 3

\begin{tabular}{lrr}
\hline Multipole & Normal & Skew \\
\hline 0 & -3.1355 & $0.0000 \mathrm{E}+00$ \\
1 & 50.0889 & $3.3886 \mathrm{E}-14$ \\
2 & 399.8327 & $1.7819 \mathrm{E}-14$ \\
3 & 0.6684 & $-2.5828 \mathrm{E}-15$ \\
4 & $-1.6765 \mathrm{E}-03$ & $-4.9361 \mathrm{E}-16$ \\
5 & $1.2598 \mathrm{E}-05$ & $1.6598 \mathrm{E}-16$ \\
6 & $-6.3185 \mathrm{E}-08$ & $1.1290 \mathrm{E}-16$ \\
7 & $5.2769 \mathrm{E}-10$ & $7.0503 \mathrm{E}-18$ \\
8 & $-3.3077 \mathrm{E}-12$ & $1.0255 \mathrm{E}-18$ \\
9 & $2.9008 \mathrm{E}-14$ & $2.9487 \mathrm{E}-19$ \\
10 & $-2.0365 \mathrm{E}-16$ & $5.3837 \mathrm{E}-20$ \\
11 & $1.8311 \mathrm{E}-18$ & $4.97717 \mathrm{E}-21$ \\
\hline
\end{tabular}

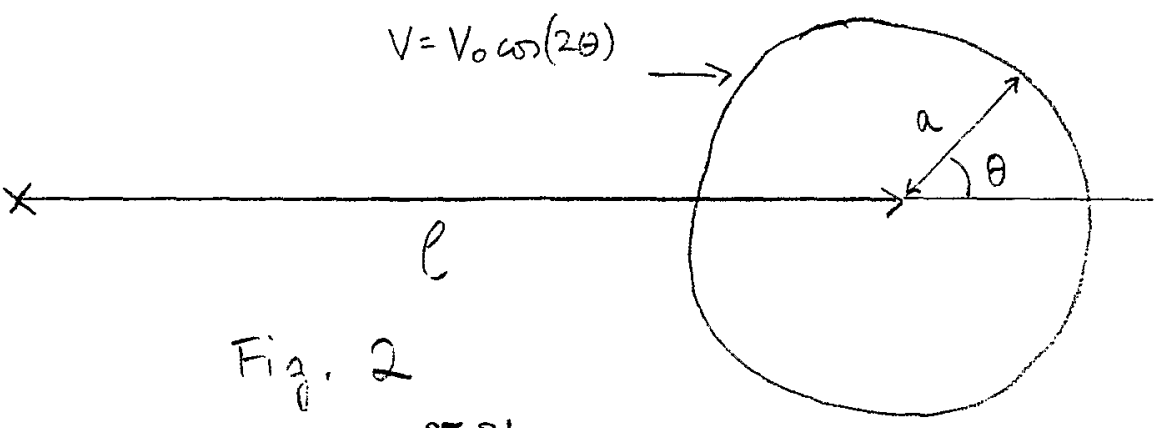




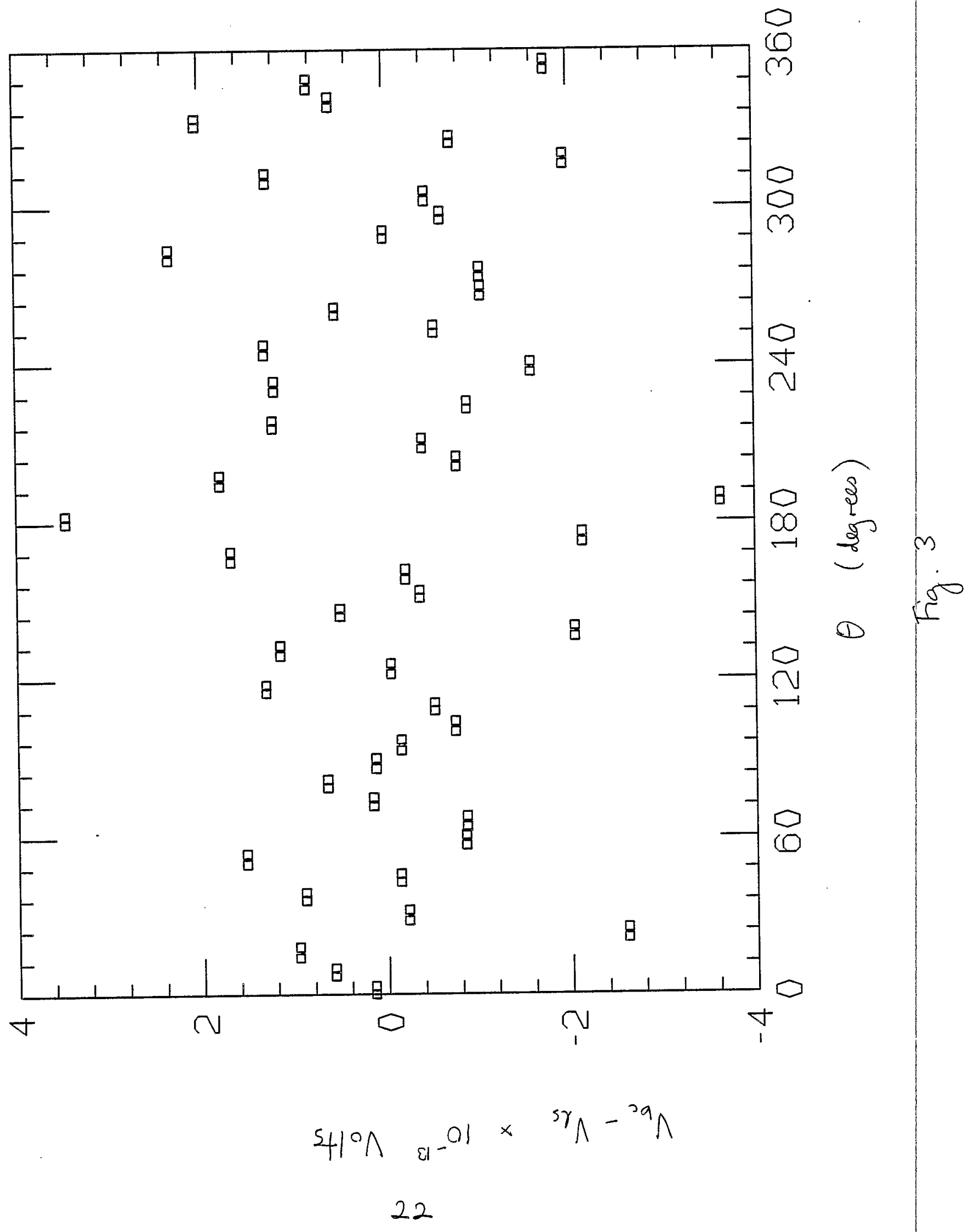




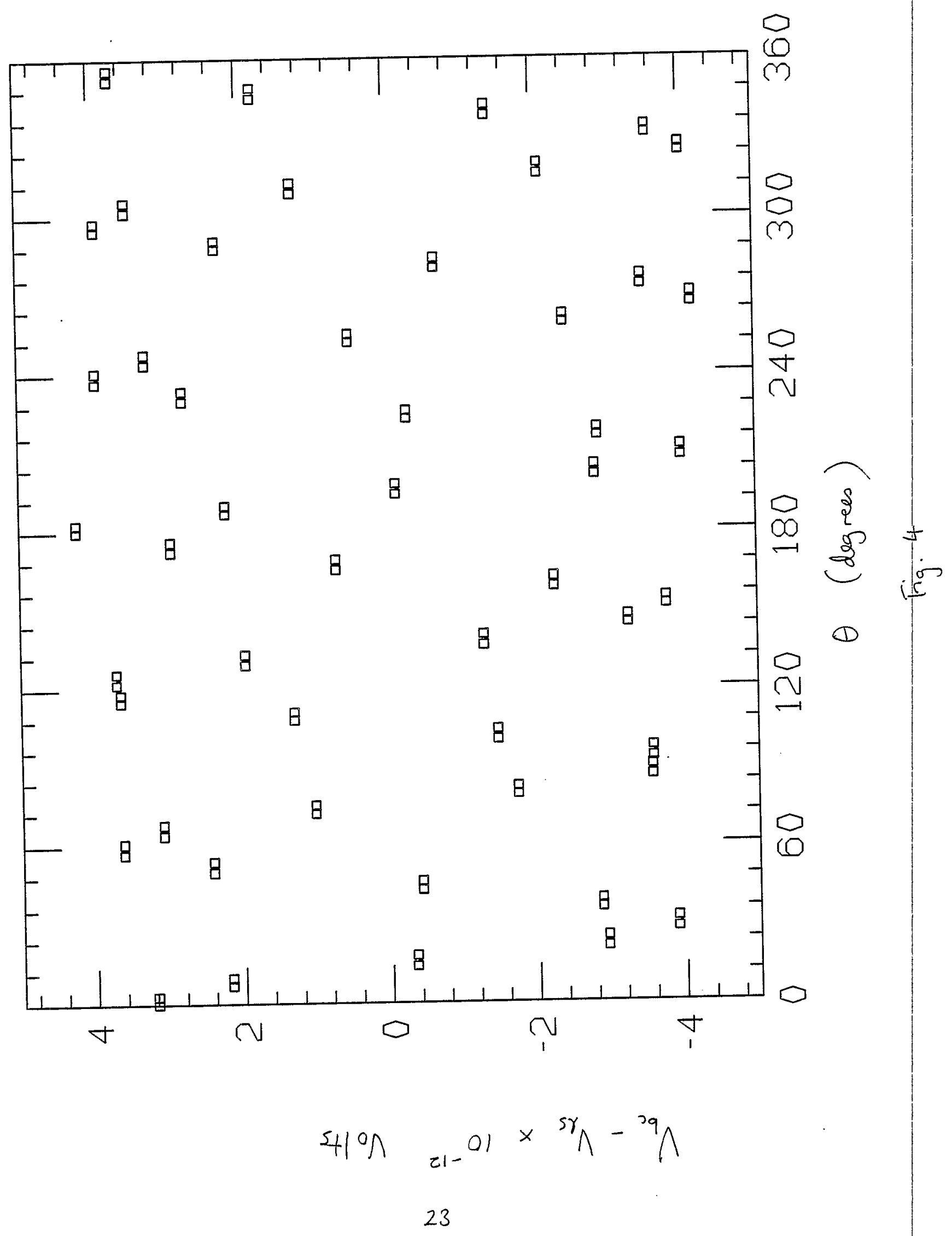




\subsubsection{Quadrupole Plates in Vacuum Chamber}

Let us now consider a more realistic example, and one where we do not know the exact answer even when the $z$ axis is straight. Consider a vacuum chamber of square cross-section, with four plates inside, as sketched in Fig. 5 . The potential is $V_{0}$ on the top and bottom plates and $-V_{0}$ on the right and left plates, where $V_{0}=10^{4}$ Volts, and the vacuum chamber is at ground.

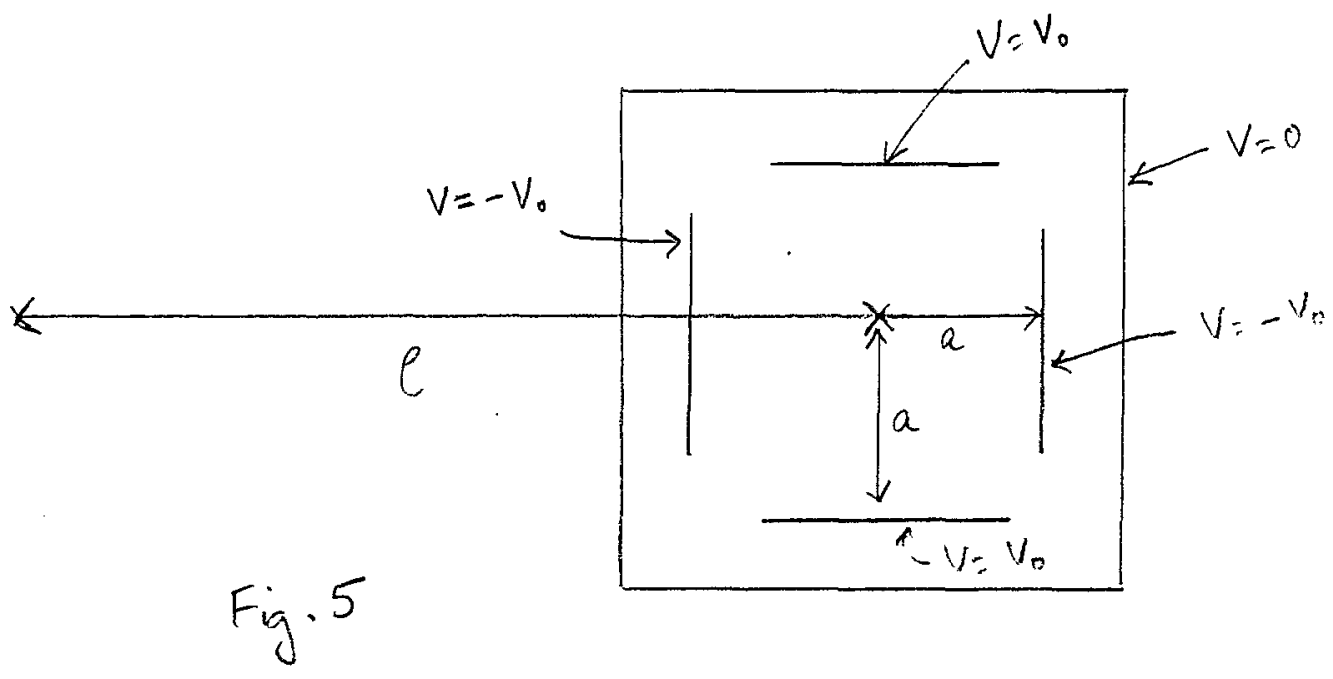

The setup forms an electrostatic quadrupole. However, there will be higher order terms present, even when the $z$ axis is straight. A geometry similar to this is being considered for the muon storage ring in the muon $g-2$ experiment at Brookhaven National Laboratory. We put $\rho_{0}=1000 \mathrm{~cm}$. The distance from the center of the vacuum chamber to each plate is $a=5 \mathrm{~cm}$. The plates have widths of $6 \mathrm{~cm}$ each. The "bounding surface" in this case consists of the surfaces of the plates, because we are only really interested in the region "enclosed" by the four plates. We fit to 50 points on each plate, spaced equally along the length of the plates, making $N=200$ points in all. Note that this time, the bounding surface does not completely enclose the region where we wish to calculate the potential, and furthermore, we are ignoring the wall of the vacuum chamber, which is at ground. We use twenty-nine terms in the sum, i.e. we put

$$
V=\tilde{B}_{0}+\sum_{n=1}^{14} \bar{B}_{n} \tilde{V}_{n}^{n}+\tilde{A}_{n} \tilde{V}_{n}^{s} .
$$

The values of the coefficients are shown in Table 4. In Fig. 6, we plot $V_{b c}-V_{l s}$ on the right, top, left and bottom plates, for 200 points spaced equally along each electrode, so $i=1,2, \ldots, 200$ on the horizontal axis denotes the points on the right electrode, $i=201,202, \ldots, 400$ denotes the points on the top electrode, and so on for the others. We see that this time $\left|V_{b c}-V_{l s}\right|$ oscillates with an amplitude of about 5 Volts, and we also see a Gibbs-like phenomenon at the edges of the plates, which is to be expected since the potential changes abruptly there. The agreement with the boundary conditions is still good, although less impressive than in the previous examples. 
Table 4

\begin{tabular}{lrr}
\hline Multipole & Normal & Skew \\
\hline 0 & $-2.0808 \mathrm{E}-02$ & 0.0000 \\
1 & -3.3759 & $1.6612 \mathrm{E}-07$ \\
2 & -437.1907 & $-2.1979 \mathrm{E}-10$ \\
3 & $-2.1346 \mathrm{E}-02$ & $5.9136 \mathrm{E}-09$ \\
4 & $-5.6687 \mathrm{E}-06$ & $-8.0756 \mathrm{E}-13$ \\
5 & $1.0032 \mathrm{E}-03$ & $-1.6011 \mathrm{E}-10$ \\
6 & $6.7347 \mathrm{E}-02$ & $1.7676 \mathrm{E}-13$ \\
7 & $8.0814 \mathrm{E}-06$ & $-4.2799 \mathrm{E}-12$ \\
8 & $2.7864 \mathrm{E}-09$ & $3.8577 \mathrm{E}-16$ \\
9 & $-2.2020 \mathrm{E}-07$ & $1.1293 \mathrm{E}-13$ \\
10 & $-1.4123 \mathrm{E}-05$ & $-7.3887 \mathrm{E}-17$ \\
11 & $-1.8813 \mathrm{E}-09$ & $4.0190 \mathrm{E}-15$ \\
12 & $-2.9885 \mathrm{E}-13$ & $3.6831 \mathrm{E}-19$ \\
13 & $5.2551 \mathrm{E}-11$ & $-7.8707 \mathrm{E}-18$ \\
14 & $3.0395 \mathrm{E}-09$ & $6.6519 \mathrm{E}-20$ \\
\hline & & \\
\hline & &
\end{tabular}

There are some obvious limitations as to how good a fit we can expect, the most notable of which are that we have ignored the outside wall (the vacuum chamber) in the fit, and the bounding surface does not fully enclose the domain of the multipole expansion. Hence the solution in this case is only valid if the outside walls (which are at ground) are far away from the electrodes, otherwise they will strongly perturb the potential in the gaps between the electrodes. Hence we cannot expect to fit the boundary conditions to as good a level as in the previous models. In particular, we should divide the entire interior region of the vacuum chamber into sections, with a different multipole expansion in each region, and fit them all simultaneously to the total boundary conditions. A program such as POISSON can solve Laplace's equation for such a system. Hence one can, if desired, use POISSON to calculate the potential as a function of $(x, y)$ coordinates, i.e. $V_{\text {poisson }}(x, y)$, and then fit the sum of curved multipoles to this function, where the $\left(x_{i}, y_{i}\right)$ points need not be on the surfaces of the electrodes anymore, but can be distributed throughout the domain of the multipole expansion. This is analogous to the use of a sum of multipoles to fit to experimental magnetic field measurements in a magnet, as described above. 


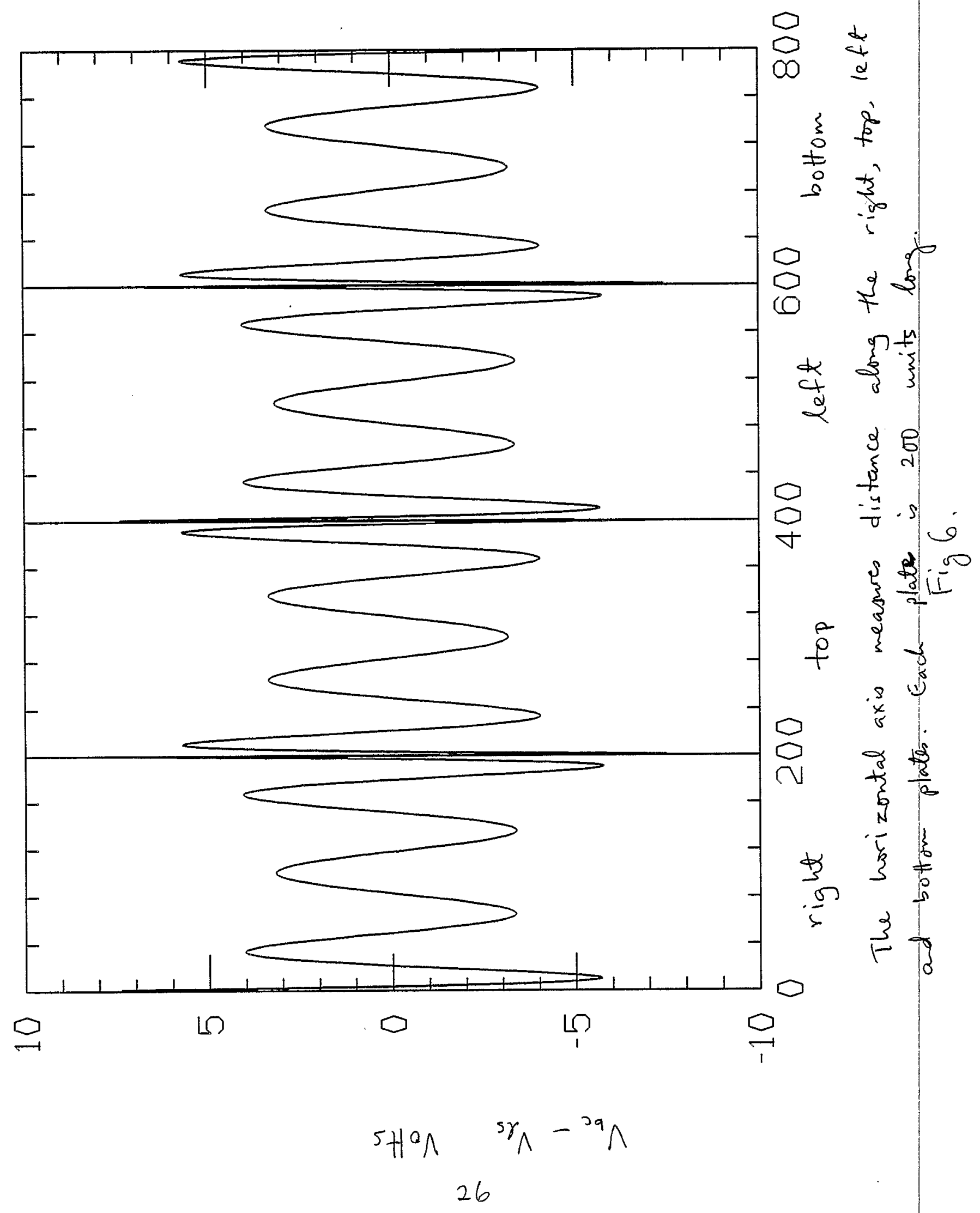




\section{Conclusion}

I have described a set of functions, which I have called curved multipoles, to generalize the straight multipoles, or Fourier harmonics, $r^{n} \cos (n \theta)$ and $r^{n} \sin (n \theta)$. The new functions satisfy Laplace's equation in two dimensions when the third or $z$ axis is curved in a circle of radius $\rho_{0}$. Hence the functions depend on $x, y$ and $\rho_{0}$. I have given curved multipoles for both the electrostatic scalar potential $V$ and the magnetic vector potential $A_{\phi}$, and also the components of the electric and magnetic fields $E_{x}, E_{y}, B_{x}$, and $B_{y}$. Finally, I described how to use these functions to fit the boundary conditions in a few model examples, to demonstrate the use of these functions. 


\section{Appendix}

\section{A Alternative Formalisms}

\section{A.1 Homogenous Polynomials}

Dragt and co-workers [1] have treated the problem of a combined function dipole, hence they solve for the vector potential $A_{\phi}$. The reference axis is curved in a circular arc, hence the quadrupole and sextupole, etc. components of the field will induce higher order terms due to the curvature. Their method is briefly described below.

Their starting equation is

$$
\left[\frac{\partial^{2}}{\partial \rho^{2}}+\frac{\partial^{2}}{\partial y^{2}}-\frac{1}{\rho} \frac{\partial}{\partial \rho}\right]\left(\rho A_{\phi}\right)=0,
$$

and new variables $\xi$ and $\eta$ are introduced via $\rho=\rho_{0}+\xi$ and $y=\eta$. Further, define $f(\xi, \eta)=\rho A_{\phi}$. From this we obtain

$$
\left[\left(\rho_{0}+\xi\right)\left(\frac{\partial^{2}}{\partial \xi^{2}}+\frac{\partial^{2}}{\partial \eta^{2}}\right)-\frac{\partial}{\partial \xi}\right] f=0 \text {. }
$$

Following Dragt et al. [1], $f$ is expanded in homogenous polynomials $U_{n}$ of degree $n$ in $\xi$ and $\eta$, via

$$
f=\sum_{n} U_{n}(\xi, \eta)
$$

The $U_{n}$ 's here are not related to the $U_{n}(x)$ functions in the main body of the text. Substituting into Eq. (A.2), and equating terms of like degree yields

$$
\left(\frac{\partial^{2}}{\partial \xi^{2}}+\frac{\partial^{2}}{\partial \eta^{2}}\right) U_{n+1}=\frac{1}{\rho_{0}}\left[\frac{\partial}{\partial \xi}-\xi\left(\frac{\partial^{2}}{\partial \xi^{2}}+\frac{\partial^{2}}{\partial \eta^{2}}\right)\right] U_{n}
$$

Next, we decompose

$$
U_{n}=P_{n}+S_{n}
$$

where $P_{n}$ is a particular solution of Eq. (A.4), to be determined below, and $S_{n}$ is a solution of the homogenous equation

$$
\left(\frac{\partial^{2}}{\partial \xi^{2}}+\frac{\partial^{2}}{\partial \eta^{2}}\right) S_{n}=0 .
$$

Thus $S_{n}=\bar{a}_{n} r^{n} \sin (n \theta)+\bar{b}_{n} r^{n} \cos (n \theta)$, where $\bar{a}_{n}$ and $\bar{b}_{n}$ are constants, and $\xi=r \cos \theta$ and $\eta=$ $r \sin \theta$. Hence $S_{n}$ is the general solution in the absence of curvature, and $P_{n}$ contains the extra terms induced by the nonzero curvature of the $z$ axis.

The calculation of $P_{n}$ is described in Ref. [1]. Starting from, say, the quadrupole

$$
S_{2}=-\frac{\bar{b}_{2} \rho_{0}}{2}\left(\xi^{2}-\eta^{2}\right)+\frac{\alpha^{2} \rho_{0}}{2}(2 \xi \eta)
$$


we find, from Eq. (A.4),

$$
\left(\frac{\partial^{2}}{\partial \xi^{2}}+\frac{\partial^{2}}{\partial \eta^{2}}\right) P_{3}=-\bar{b}_{2} \xi+\bar{a}_{2} \eta
$$

A particular solution for this is given by

$$
\begin{aligned}
P_{3} & =-\frac{\bar{b}_{2}}{8} \xi\left(\xi^{2}+\eta^{2}\right)+\frac{\bar{a}_{2}}{8} \eta\left(\xi^{2}+\eta^{2}\right) \\
& =-\frac{\bar{b}_{2}}{8} r^{3} \cos \theta+\frac{\bar{a}_{2}}{8} r^{3} \sin \theta .
\end{aligned}
$$

The solution is chosen so that $P_{3}$ has no term that varies like $3 \theta$. This makes it orthogonal to $S_{3}$. In general, we choose $P_{n}$ such that it will have no term which varies like $n \theta$, hence it will be orthogonal to $S_{n}$. This criterion makes the solution for $P_{n}$ unique. For example, putting

$$
S_{3}=-\frac{\bar{b}_{3} \rho_{0}}{3}\left(\xi^{3}-3 \xi \eta^{2}\right)+\frac{\bar{a}_{3} \rho_{0}}{3}\left(3 \xi^{2} \eta-\eta^{3}\right)
$$

yields

$$
\left(\frac{\partial^{2}}{\partial \xi^{2}}+\frac{\partial^{2}}{\partial \eta^{2}}\right) P_{4}=-\xi^{2}\left(\bar{b}_{3}-\frac{5 \bar{b}_{2}}{8 \rho_{0}}\right)+\xi \eta\left(2 \bar{a}_{3}-\frac{3 \bar{a}_{2}}{4 \rho_{0}}\right)+\eta^{2}\left(\vec{b}_{3}-\frac{\bar{b}_{2}}{8 \rho_{0}}\right)
$$

the solution for which is

$$
\begin{aligned}
P_{4} & =\frac{\bar{b}_{2}}{64 \rho_{0}}\left(\xi^{2}+\eta^{2}\right)^{2}-\left(\frac{\bar{b}_{3}}{12}-\frac{\bar{b}_{2}}{32 \rho_{0}}\right)\left(\xi^{4}-\eta^{4}\right)+\left(\frac{\bar{a}_{3}}{6}-\frac{\bar{a}_{2}}{16 \rho_{0}}\right) \xi \eta\left(\xi^{2}+\eta^{2}\right) \\
& =\frac{\bar{b}_{2}}{64 \rho_{0}} r^{4}-\left(\frac{\bar{b}_{3}}{12}-\frac{\bar{b}_{2}}{32 \rho_{0}}\right) r^{4} \cos (2 \theta)+\left(\frac{\bar{a}_{3}}{12}-\frac{\bar{a}_{2}}{32 \rho_{0}}\right) r^{4} \sin (2 \theta) .
\end{aligned}
$$

We see that $P_{4}$ has no term which varies like $4 \theta$. One can continue this to derive expressions for $P_{5}, P_{6}$, etc. up to any desired order.

\section{A.2 Two-Dimensional Power Series}

A method of calculating the coefficients for a scalar potential, when the $z$ axis is curved, has also been given by Berz [2], who includes the possibility of $z$ dependence in the potential. The method is briefly described here. As pointed out by Berz [2], the method is also applicable to magnetostatic problems, since $\vec{\nabla} \cdot \vec{B}=\vec{\nabla} \times \vec{B}=0$, hence $\vec{B}$ can be derived from a scalar potential. We start with Eq. (2.9), and put

$$
V=\sum_{m=0}^{\infty} \sum_{n=0}^{\infty} a_{m n}(z) \frac{x^{m} y^{n}}{m ! n !} .
$$

Then, using primes to denote derivatives with respect to $z$,

$$
\frac{\partial V}{\partial z}=\sum_{m n} a_{m n}^{\prime} \frac{x^{m} y^{n}}{m ! n !}, \quad \frac{\partial^{2} V}{\partial z^{2}}=\sum_{m n} a_{m n}^{\prime \prime} \frac{x^{m} y^{n}}{m ! n !},
$$


and so

$$
\frac{\partial}{\partial z}\left(\frac{1}{1+h x} \frac{\partial V}{\partial z}\right)=\frac{1}{1+h x} \frac{\partial^{2} V}{\partial z^{2}}-\frac{h^{\prime} x}{(1+h x)^{2}} \frac{\partial V}{\partial z} .
$$

We substitute this into Eq. (2.9), and multiply throughout by $(1+h x)^{3}$, which yields

$$
\begin{aligned}
0 & =(1+h x)^{2} \frac{\partial}{\partial x}\left[(1+h x) \frac{\partial V}{\partial x}\right]+(1+h x)^{3} \frac{\partial^{2} V}{\partial y^{2}}+(1+h x) \frac{\partial^{2} V}{\partial z^{2}}-h^{\prime} x \frac{\partial V}{\partial z} \\
& =(1+h x)^{3} \frac{\partial^{2} V}{\partial x^{2}}+h(1+h x)^{2} \frac{\partial V}{\partial x}+(1+h x)^{3} \frac{\partial^{2} V}{\partial y^{2}}+(1+h x) \frac{\partial^{2} V}{\partial z^{2}}-h^{\prime} x \frac{\partial V}{\partial z} .
\end{aligned}
$$

Hence

$$
\begin{aligned}
0= & \left(1+3 h x+3 h^{2} x^{2}+h^{3} x^{3}\right) \sum_{m n} a_{m n} \frac{x^{m-2} y^{n}}{(m-2) ! n !} \\
& +h\left(1+2 h x+h^{2} x^{2}\right) \sum_{m n} a_{m n} \frac{x^{m-1} y^{n}}{(m-1) ! n !} \\
& +\left(1+3 h x+3 h^{2} x^{2}+h^{3} x^{3}\right) \sum_{m n} a_{m n} \frac{x^{m} y^{n-2}}{m !(n-2) !} \\
& +(1+h x) \sum_{m n} a_{m n}^{\prime \prime} \frac{x^{m} y^{n}}{m ! n !}+h^{\prime} x \sum_{m n} a_{m n}^{\prime} \frac{x^{m} y^{n}}{m ! n !} .
\end{aligned}
$$

We collect terms in $x^{m} y^{n}$, and multiply by $m ! n !$, which yields

$$
\begin{aligned}
a_{m n+2}=- & a_{m n}^{\prime \prime}-m h_{m-1 n}^{\prime \prime}+m h^{\prime} a_{m-1 n} \\
- & -\left[a_{m+2 n}+(3 m+1) h a_{m+1 n}+3 m h a_{m-1 n+2}\right. \\
& +m(3 m-1) h^{2} a_{m n}+3 m(m-1) h^{2} a_{m-2 n+2} \\
& \left.\quad+m(m-1)^{3} h^{3} a_{m-1 n}+m(m-1)(m-2) h^{3} a_{m-3 n+2}\right],
\end{aligned}
$$

which can be used to calculate $a_{m+2}$ in terms of other coefficients with smaller values of $m$ and/or $n$. It is understood that a coefficient vanishes if either of its indices takes a negative value.

The above recursion relation simplifies considerably if the potential, and the curvature $h$, are independent of $z$. Then we use Eq. (2.10), and we find

$$
a_{m n+2}=-a_{m+2 n}-(m+1) h a_{m+1 n}-m h a_{m-1 n+2} .
$$

This method will yield a solution for $V$ as a sum of terms of the form $x^{m} y^{n}$, i.e. a polynomial or power series in two variables, as opposed to a multipole-type expansion. One can, if desired, group the terms so that $m+n$ adds up to a constant within each set, i.e.

$$
V=\sum_{k=0}^{\infty} \sum_{n=0}^{k} a_{n k-n} \frac{x^{n} y^{k-n}}{n !(k-n) !}
$$


in which case $V$ will be a sum of homogenous polynomials of degree $k,(=0,1,2, \ldots)$ in $x$ and $y$. In the limit of zero curvature, $h \rightarrow 0$, the polynomials will reduce to the usual multipoles, as listed in Eq. (2.3).

\section{References}

[1] A.J. Dragt et al., Univ. of Maryland preprint PP 90-074, and submitted to Nucl. Instr. and Meth.

[2] M. Berz, Nucl. Instr. and Meth. A298 (1990) pp. 473-479. 